\title{
Ultrafast large-scale simulations of biophysically realistic neurons using deep learning
}

\author{
Viktor Janos Olah ${ }^{1}$, Nigel P Pedersen ${ }^{2} \&$ Matthew JM Rowan ${ }^{1 *}$ \\ ${ }^{1}$ Department of Cell Biology, Emory University School of Medicine, Atlanta, GA, 30322, USA \\ ${ }^{2}$ Department of Neurology, Emory University School of Medicine, Atlanta, GA, 30322, USA
}

\author{
*Address for Correspondence: \\ Matt Rowan, $\mathrm{PhD}$ \\ Emory University School of Medicine \\ Department of Cell Biology \\ Atlanta GA, 30322 \\ Email: mjrowan@emory.edu
}

\begin{abstract}
Understanding the activity of the mammalian brain requires an integrative knowledge of circuits at distinct scales, ranging from ion channel time constants to synaptic connection probabilities. To understand how multiple parameters contribute synergistically to circuit behavior as a whole, neuronal computational models are regularly employed. However, traditional models containing anatomically and biophysically realistic neurons are computationally expensive when scaled to model local circuits. To overcome this limitation, we trained several artificial neural net (ANN) architectures to model the activity of realistic, multicompartmental neurons. We identified a single ANN that accurately predicted both subthreshold and action potential firing, and correctly generalized its responses to previously unobserved synaptic input. When scaled, processing times were orders of magnitude faster compared with traditional approaches, allowing for rapid parameterspace mapping in a circuit model of Rett syndrome. Thus, our ANN approach allows for rapid, detailed network experiments using inexpensive, readily available computational resources.
\end{abstract}

\section{Introduction}

Understanding the behavior of complex neural circuits like the human brain is one of the fundamental challenges of this century. Predicting mammalian circuit behavior is difficult due to several different underlying mechanisms at distinct organizational levels, ranging from molecular-level interactions to large-scale connection motifs. Computational modeling has become a cornerstone technique for deriving and testing new hypotheses about brain organization and function ${ }^{1-4}$. In little more than 60 years, our mechanistic understanding of neural function has evolved from describing action potential (AP) related ion channel gating ${ }^{5}$ to constructing models that can simulate the activity 
of whole brain regions ${ }^{6-10}$. Although tremendous advancements have been made in the development of computational resources, the lack of available or affordable hardware for neural simulations currently represents a significant barrier to entry for most neuroscientists and renders many questions intractable. This is particularly well illustrated by large-scale neural circuit simulations. In contrast to detailed single-cell models, which have been a regular occurrence in publications since the '90s ${ }^{11-18}$, parallel simulation of thousands, or even hundreds of thousands of detailed neurons have only become a possibility with the advent of supercomputers ${ }^{19-26}$. As these resources are still not widely accessible, several attempts have been made to mitigate the immense computational load of large-scale neural simulations by judicious simplification ${ }^{19,27-33}$, however, simplifications inevitably result in feature or information loss, such as sacrificing multicompartmental information for simulation speed ${ }^{19,27,28,30}$. Thus, there is a critical need for large-scale neural simulations which incorporate arbitrary features of individual neuronal cells, such as dendritic information processing or specific ionic currents, that are also fast enough to be simulated on widely available computational resources.

To counteract the increasing computational burden of ever-growing datasets on more traditional models, many different fields have recently adopted various machine learning algorithms ${ }^{34-}$ ${ }^{38}$. Specifically, artificial neural networks (ANNs) have been shown to be superior to traditional model systems both in terms of speed and accuracy when dealing with complex systems such as those governing global financial markets or weather patterns ${ }^{39,40}$. Due to their accelerated processing speed, ANNs are ideal candidates for modeling large-scale biological systems. The idea that individual neural cells could be represented by ANNs was proposed almost two decades ago ${ }^{41}$, however conclusive evidence for the feasibility of such a model is lacking (but see ${ }^{42}$ ). Therefore, our aim was to develop an ANN which can (1) accurately replicate various features of biophysically detailed neuron models, (2) efficiently generalize for previously unobserved input conditions and (3) significantly accelerate large-scale network simulations.

Here we investigated several ANN architectures to understand which most accurately represented membrane potential dynamics, in both simplified point neurons as well as multicompartment neurons. Among the selected ANNs, we found that a convolutional recurrent architecture can simulate both subthreshold and suprathreshold voltage dynamics. Furthermore, this ANN could generalize to a wide range of input conditions and could reproduce neuronal features following different input patterns beyond membrane potential responses, such as ionic current waveforms. Next, we demonstrated that this ANN can also accurately predict multicompartmental information, by fitting this architecture to a biophysically detailed layer 5 pyramidal cell ${ }^{43}$ model. Importantly, we found that ANN representations could exponentially accelerate large network 
simulations, as demonstrated by network parameter space mapping of a cortical layer 5 recurrent microcircuit model of Rett syndrome, a neurodegenerative disorder that is associated with cortical dysfunction and epilepsy ${ }^{44-47}$. Thus, we provide a detailed description of an ANN architecture suitable for large scale simulations of anatomically and biophysically detailed neurons, that has applicability to human disease modelling. Most importantly, our ANN simulations are accelerated to the point where detailed network experiments can now be carried out using inexpensive, readily available computational resources.

\section{Results}

To create a deep learning platform capable of accurately representing the full dynamic membrane potential range of neuronal cells, we focused on model systems proven to be suitable for multivariate time series forecasting (MTSF). MTSF models are potentially ideal for this purpose, as they can be designed to receive information about past synaptic inputs and membrane potentials in order to predict subsequent voltage responses. These ANNs have recently been demonstrated to be superior to other algorithms in handling multivariate temporal data such as audio signals ${ }^{48}$, natural language $\mathrm{H}^{49}$ and various other types of fluctuating time series datasets ${ }^{50-52}$. Whether these models are capable of accurately predicting the full range of activity patterns in excitable cells is unclear.

Although deep learning approaches are now well established in various disciplines, the need to incorporate ANNs in different elements of neuroscience research is evident ${ }^{53}$. Due to the recent surge in the availability of various large datasets, the utility of ANN architecture customization for different purposes is increasingly appreciated ${ }^{54-57}$. To compare the ability of different ANNs to reproduce the activity of an excitable cell, we designed five distinct architectures (Figure 1A). The first two models were a simple linear model with one hidden layer (linear model, Figure 1A, blue) and a similar model equipped with nonlinear processing (nonlinear model, Figure 1A, cyan), as even relatively simple model architectures can explain the majority of subthreshold membrane potential variance $^{58}$. The third and fourth models consist of recently constructed time-series forecasting architectures, including a recurrent ANN (CNN-LSTM, Figure 1A, magenta) consisting of convolutional layers ${ }^{49}$, long short-term memory $\left(\operatorname{LSTM}^{59,60}\right)$ layers, and fully connected layers, termed the CNN-LSTM network (Supplementary Figure $1,{ }^{61}$ ) and a more recently developed architecture 


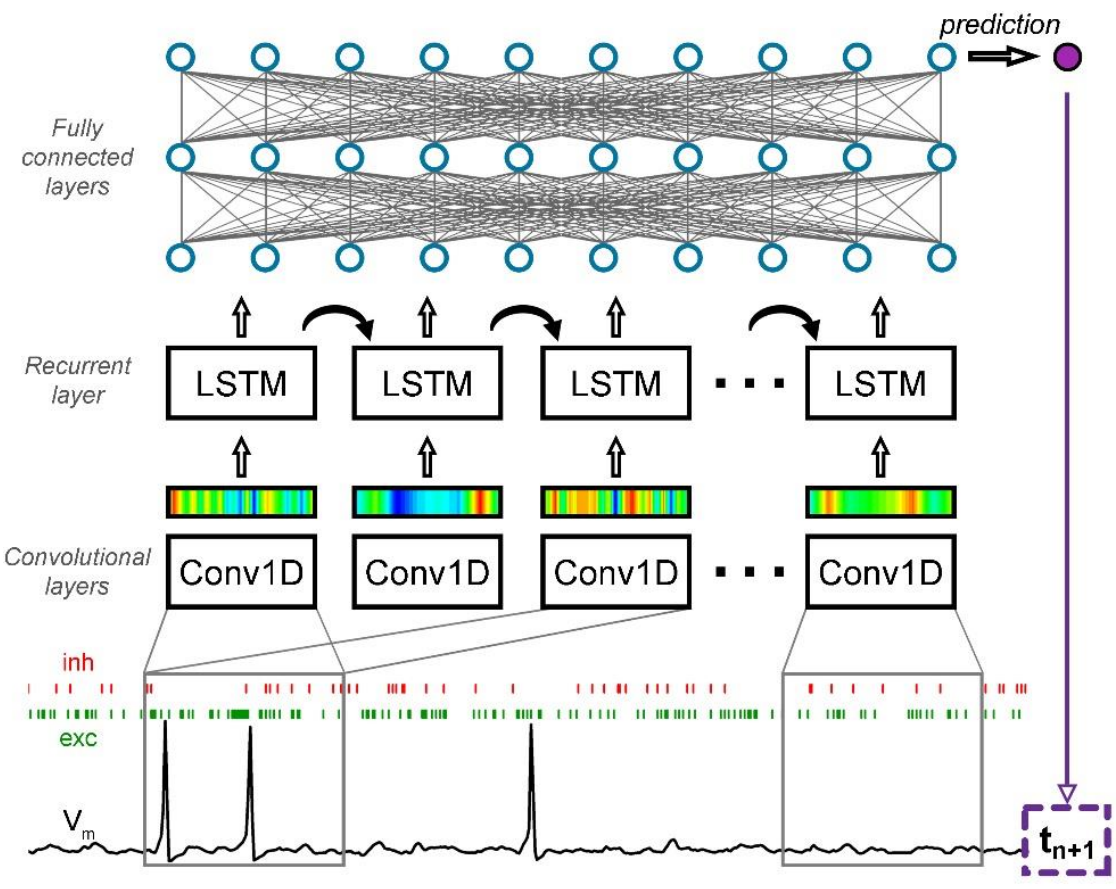

Supplementary Figure 1. CNN-LSTM architecture for time series forecasting. The input of the ANN consisted of a membrane potential vector $\left(V_{m}\right)$ and the weights and onsets of synaptic inputs (a representative inhibitory synapse - inh in red, and an excitatory synapse - exc in green). The first layer of the ANN (Conv1D) creates a temporally alligned convolved representation (colored bars) of the input by sliding a colvolutional kernel (grey box) along the input. The second functional block (LSTM layers) processes the output of the convolutional layers through recurrent connections, to weigh information temporally. The last functional block consisting of fully connected layers provides additional nonlinear information processing power. The output of the network in this case is the first subsequent $V_{m}$ value $\left(t_{n+1}\right)$. The number of layers belonging to specific functional blocks of the CNN-LSTM architecture may vary. relying on dilated temporal convolutions (convolutional net, Figure 1A, orange) (based on the WaveNet $\operatorname{architecture}^{42,62}$ ), which is superior to the CNN-LSTM in several MTSF tasks. The CNN-LSTM has the distinct advantage of having almost two orders of magnitude more adjustable parameters compared to the aforementioned ANNs. Finally, we selected a fifth architecture (deep neural net, Figure 1A, green) with a comparable number of free parameters to the $\mathrm{CNN}$ LSTM, composed of ten hidden layers, and which

operates solely on linear and nonlinear transformations. Before moving to neural cell data, each of the five selected architectures were evaluated using a well-curated weather time series dataset (see methods). Each model performed similarly (0.070/0.069, 0.059/0.06, 0.089/0.094, 0.07/0.069, 0.092/0.095, mean absolute error on the validation/testing datasets for linear, nonlinear, convolutional net and CNN-LSTM, deep neural net architectures, respectively), demonstrating their suitability for MTSF problems.

\section{Prediction of point neuron membrane potential dynamics by ANNs}

To test the ability of the five ANNs to represent input-output transformations of a neural cell, we next fitted these architectures with data from passive responses of a single-compartmental pointneuron model (NEURON simulation environment ${ }^{63}$ ) using the standard backpropagation learning algorithm for $\mathrm{ANNs}^{64}$. Each model was tasked with predicting a single membrane potential value based 
bioRxiv preprint doi: https://doi.org/10.1101/2021.02.22.432356; this version posted February 23, 2021. The copyright holder for this preprint (which was not certified by peer review) is the author/funder, who has granted bioRxiv a license to display the preprint in perpetuity. It is made available under aCC-BY-NC-ND 4.0 International license.
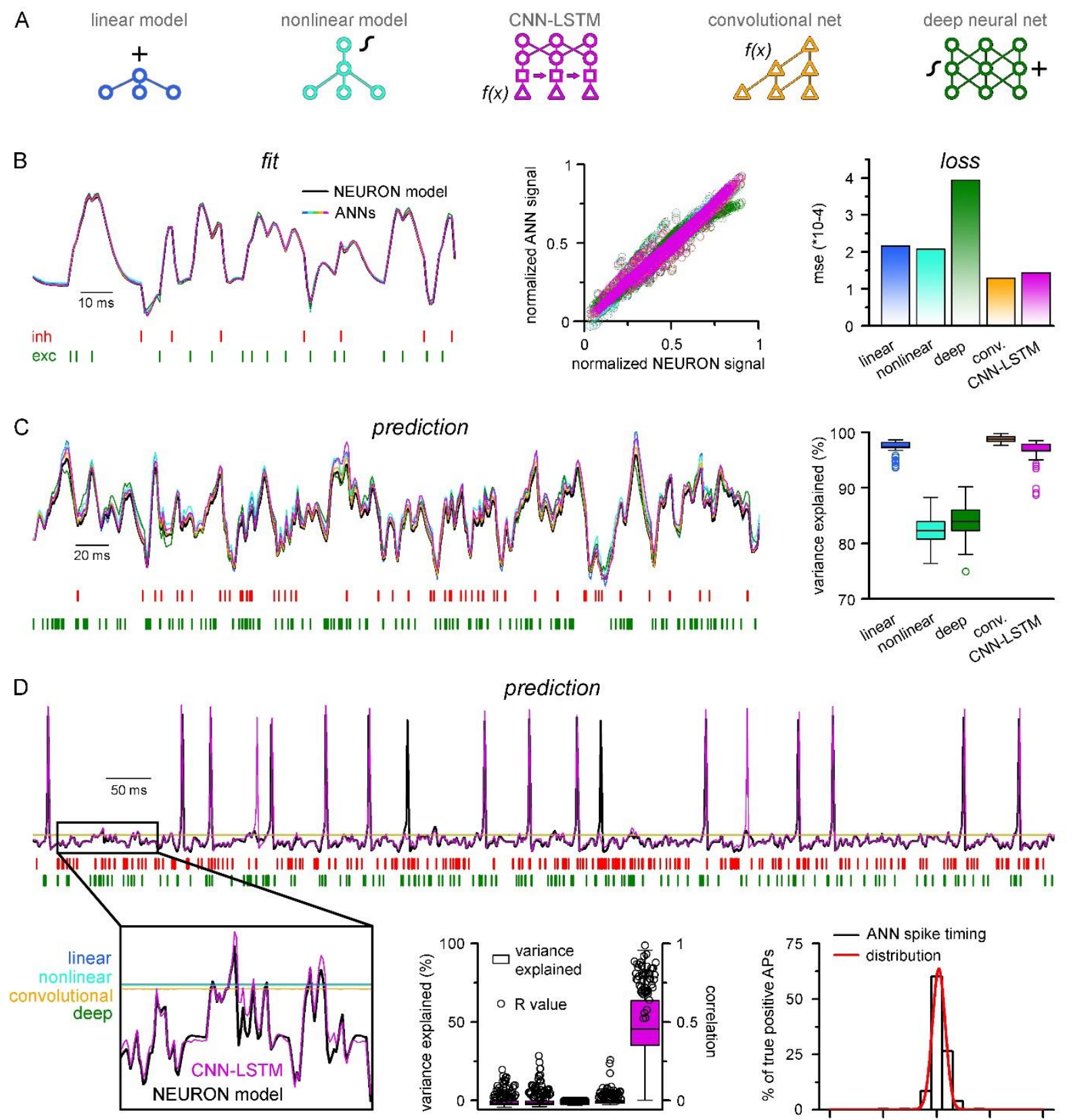

\section{prediction}


quantal size: $2.5 \mathrm{nS}$ for excitatory, $8 \mathrm{nS}$ for inhibitory inputs) sampled at $1 \mathrm{kHz}$. We found that both the linear and nonlinear models predicted subsequent membrane potential values with low error rates (Figure 1B) with similar behavior in both the CNN-LSTM and convolutional architectures $\left(2.16^{*} 10^{-4}\right.$ $\pm 1.18 * 10^{-3}, 2.07 * 10^{-4} \pm 1.11 * 10^{-3}, 1.43 * 10^{-4} \pm 9.31 * 10^{-4}, 1.29 * 10^{-4} \pm 9.42 * 10^{-4}$ mean error for linear, nonlinear, CNN-LSTM and convolutional models, respectively). However, the deep neural network performed considerably worse than all other tested models $\left(3.94 * 10^{-4} \pm 1.56 * 10^{-3}\right.$ mean error), potentially due to the nonlinear correspondence of its predicted values to the ground truth data (Figure 1B).

Next, we tested ANNs in simulation conditions similar to the traditional models. To this end, we initialized ANNs with ground truth data followed by a continuous query period in which forecasted membrane potential values were fed back to the ANNs to observe continuous unconstrained predictions. As expected from the fit error rates of single membrane potential forecasting (Figure 1B), continuous predictions of the linear, convolutional, and CNN-LSTM models were able to explain the ground truth signal variance at high accuracy while the deep neural net performed slightly worse (Figure 1C, $0.97 \pm 0.01 \%, 0.99 \pm 0.01 \%, 0.97 \pm 0.02 \%, 0.84 \pm 0.03 \%$ variance explained for linear, convolutional, CNN-LSTM, and deep neural net architectures respectively, $n=50$ ). Surprisingly, the nonlinear model produced the worst prediction for passive membrane potential traces $(0.82 \pm 0.03 \%$ variance explained, $\mathrm{n}=50$ ) despite performing the best on the benchmark dataset. These results indicate that even simple linear ANNs are able to capture subthreshold membrane potential behavior accurately ${ }^{58}$.

Although subthreshold events were accurately predicted by several different ANN architectures, our aim was to test how these models perform on the full dynamic range of neural cells, which due to AP firing (which can also be viewed as highly relevant outlier datapoints) constitutes a non-normally distributed and thus demanding dataset for ANNs. To investigate how the ANNs performed with suprathreshold events included, we fitted the weights and biases of the five previously investigated ANNs on active membrane potential traces. Interestingly, we found that only the CNNLSTM architecture could precisely reproduce both subthreshold membrane potential dynamics and spiking activity, while all other tested ANNs converged to the mean of the training dataset values (Figure 1D, $-0.027 \pm 0.073 \%,-0.025 \pm 0.068 \%,-0.018 \pm 0.054 \%, 0.456 \pm 0.304 \%,-0.025 \pm 0.067 \%$ variance explained for linear, nonlinear, convolutional net and CNN-LSTM, deep neural net architectures respectively, $\mathrm{n}=50$ ). We found that although the CNN-LSTM model explained substantially less variance for the active membrane potential traces (Figure 1D) than for subthreshold voltages alone (Figure 1C) due to the overall larger variance of active signals, the predictions showed 
high linear correlation with the ground truth signals (Pearson's $r=0.76793 \pm 0.10003, n=50$ ). For the four remaining ANN architectures it is unlikely that convergence to the mean is caused by settling in local minima on the fitting error surface, as ANNs have a large number of free parameters $\left(2.07 * 10^{4}\right.$, $2.07 * 10^{4}, 2.47 * 10^{6}, 3.64 * 10^{5}, 1.95 * 10^{6}$ free parameters for linear, nonlinear, deep, convolutional ANNs and CNN-LSTM respectively), therefore the chance of having a zero derivate for each parameter at the same point is extremely low ${ }^{65}$ suggesting that erroneous fitting is the consequence of the limitations of these ANN architectures. Consequently, of the tested ANN architectures the CNN-LSTM is the only model that could depict the full dynamic range of a biophysical neural model.

Closer inspection of the timing of the predicted APs revealed that the CNN-LSTM models correctly learned thresholding, as the occurrence of the APs matched the timing of the testing dataset (Figure 1D; $83.94 \pm 16.89 \%$ precision and $90.94 \pm 12.13 \%$ recall, $0.24 \pm 0.79 \mathrm{~ms}$ temporal shift for true positive spikes compared to ground truth, $\mathrm{n}=283$ ). Taken together, we developed an ANN architecture that is ideally suited for predicting both subthreshold membrane potential fluctuations and the precise timing of APs on a millisecond timescale.

\section{Generalization of the CNN-LSTM architecture}

In order to test the applicability of the CNN-LSTM for predicting physiological cellular behavior, we assessed the generalization capability of the architecture built for active behavior prediction (Figure 1D). Generalization is the ability of an ANN to accurately respond to novel data ${ }^{66,}$ 67. This capability of neural nets is largely constrained by the amount of training received, as both under- or over-training can result in poor generalization. So far, we have only tested ANNs using input patterns which were similar to the training dataset, namely, single stochastically arriving quantal inputs with invariable quantal sizes. However, if the CNN-LSTM correctly learned the mechanistic operations of a neural cell, then the architecture should behave appropriately when tasked with responding to novel quantal input patterns or amplitudes.

We first challenged the CNN-LSTM by administration of excitatory inputs with variable quantal sizes (0.1-3.5 nS, 0.1 nS increment). Similar to the NEURON model control, the CNN-LSTM responded linearly in subthreshold voltage regimes (Figure 2A, Pearson's $r=0.99, n=35$ ) and elicited an AP after reaching threshold. Independent evaluation of the NEURON model control revealed a 
A

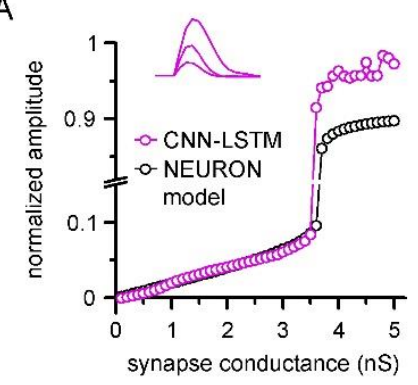

B

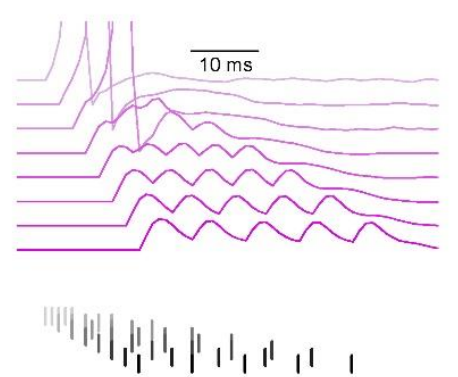

C

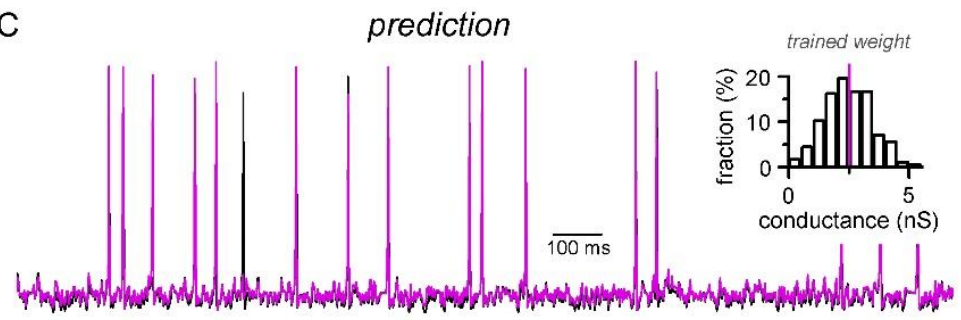

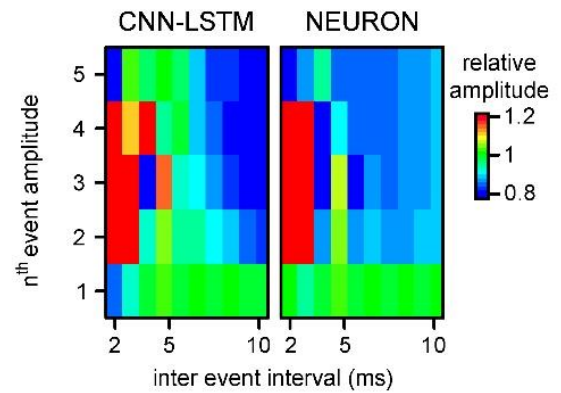

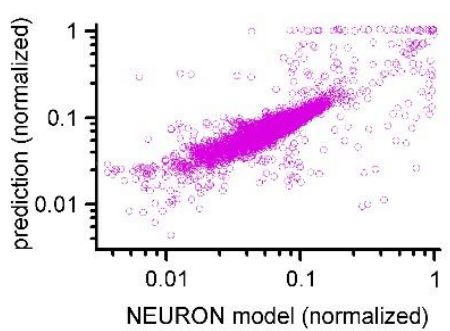

Figure 2. Ideal generalization of the CNN-LSTM trained on single input weight. A. CNN-LSTM models predict similar subthreshold event amplitudes and action potential threshold (break in y-axis) for increasing input weight, compared to NEURON models. B. CNN-LSTM models correctly represent temporal summation of synaptic events. Representative traces for different inter-event intervals (range: 2-10 ms) on the left, comparison of individual events in a stimulus train, relative to the amplitude of unitary events on the right. C. Single simulated active membrane potential trace in CNN-LSTM (purple) and NEURON (black) with variable synaptic input weights (left). The inset shows the distribution of synaptic weights used for testing generalization, with the original trained synaptic weight in purple. CNN-LSTM predicted membrane potential values plotted against NEURON model ground truth (right). Plotted values correspond to continously predicted CNN-LSTM traces.

surprisingly similar I/V relationship for the same quantal inputs (intercept, $-0.003 \pm 8.53$ and $-0.003 \pm$ 0.001; slope for subthreshold linear I/V, $22.2 \pm 0.41$ and $23.31 \pm 0.62$; CNN-LSTM and NEURON model, respectively) and similar AP threshold (-58.03 $\mathrm{mV}$ and $-56.64 \mathrm{mV}$ for CNN-LSTM and NEURON model, respectively). Next, we tested temporal summation of excitatory inputs (Figure 2B). To this end, five consecutive excitatory inputs were delivered to the same synapse with different interevent intervals (2-10 ms, $1 \mathrm{~ms}$ increments). We found that the independently simulated NEURON model displayed similar temporal summation patterns to the CNN-LSTM both for sub- and suprathreshold events (Figure 2B). Finally, we combined the previous two tests and delivered unique temporal patterns of synaptic inputs with variable synaptic conductances randomly chosen from a normal distribution (mean: $2.5 \mathrm{nS}$, variance: $0.001 \mathrm{nS}$, Figure 2C). Again, the predictions of the CNNLSTM architecture closely matched traces obtained from the NEURON model (Pearson's $r=0.81, n$ $=5000 \mathrm{~ms})$ and the timing of the majority of the APs agreed with the ground truth data $(91.02 \pm 16.03$ $\%$ recall and $69.38 \pm 22.43 \%$ precision, $\mathrm{n}=50$ ). 


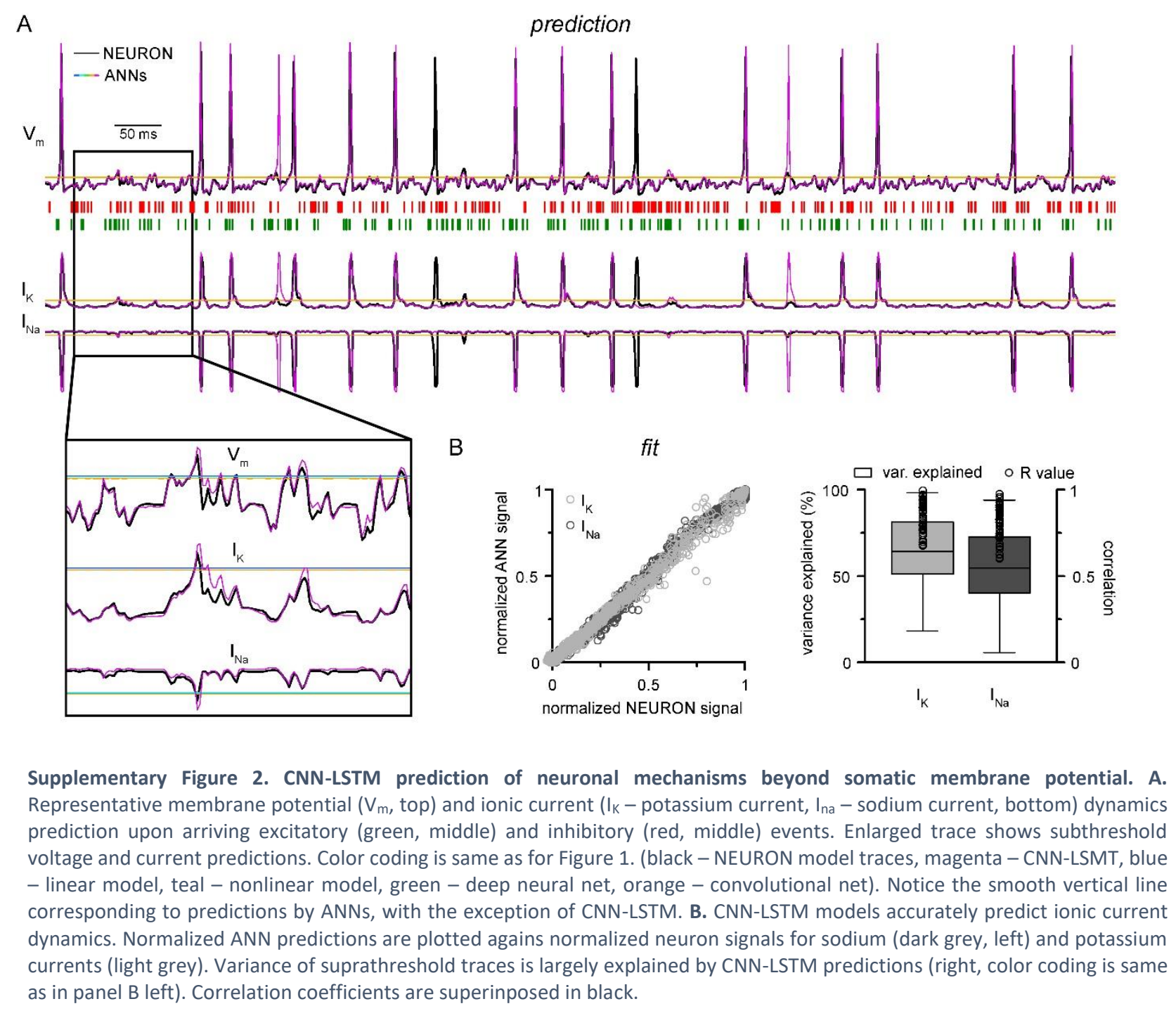

NEURON models are able to calculate and display several features of neuronal behavior in addition to membrane potential, including ionic current flux. To test how our CNN-LSTMs perform in predicting ionic current changes, we supplemented ANN inputs with sodium $\left(\mathrm{I}_{\mathrm{Na}}\right)$ and potassium currents $\left(\mathrm{I}_{\mathrm{K}}\right)$ and tasked the models to predict these values as well. The accuracy of the CNN-LSTM prediction for these ionic currents was similar to membrane potential predictions (Supplementary Fig 2, Pearson's $r=0.999$ and 0.99 for fitting, $n=5000$, variance explained: $0.64 \pm 0.21$ and $0.55 \pm 0.23$, prediction correlation coefficient: $0.85 \pm 0.08$ and $0.81 \pm 0.1, \mathrm{n}=5$, for $\mathrm{I}_{\mathrm{K}}$ and $\mathrm{I}_{\mathrm{Na}}$, respectively) while the other ANNs again regressed to the mean. Together, these results demonstrate that the CNN-LSTM correctly learned several highly specialized aspects of neuronal behavior. 


\section{Predicting the activity of morphologically realistic neurons using ANNs}

Neurons multiply their adaptive properties by segregating different conductances into separate subcellular compartments ${ }^{68-75}$. Thus, in addition to simplified input integrating point neurons, a substantial portion of neuronal models developed in recent decades intended to address subcellular signal processing via detailed multicompartmental biophysical cellular representations ${ }^{15,43,}$ 74, 76-78. Therefore, our next aim was to examine how well ANNs describe multicompartmental information. To this end, a training dataset of synaptic inputs and corresponding somatic voltage responses was generated in NEURON from a morphologically and biophysically detailed in-vivo labeled neocortical layer 5 (L5) pyramidal cell (PC) ${ }^{43}$. The simulated cell received 150 excitatory inputs (100 inputs on apical, oblique and tuft dendrites and 50 inputs on the basal dendrite, randomly distributed) and 50 inhibitory inputs (30 inputs on apical, oblique and tuft dendrites and 20 inputs on the basal dendrite, randomly distributed; Figure 3A). We found that this pyramidal cell-trained CNN-LSTM performed in near-perfect accordance with the NEURON simulation (Figure 3A, Pearson's $r=0.999, n=45000$ $\mathrm{ms}$ ). The continuous self-reliant prediction yielded lower yet adequate AP fidelity (Figure 3G, $68.28 \pm$ $18.97 \%$ and $66.52 \pm 25.37 \%$ precision and recall, $0.439 \pm 4.181 \mathrm{~ms}$ temporal shift for true positive spikes compared to ground truth, $\mathrm{n}=205$ ) compared to the point neuron, and the accuracy of subthreshold membrane potential fluctuations remained high (Pearson's $r=0.83, \mathrm{n}=37$ ).

Establishing a proper multicompartmental representation of a neural system by relying solely on the somatic membrane potential is a nontrivial task due to complex signal processing mechanisms taking place in distal subcellular compartments ${ }^{79-83}$. This is especially true with respect to signals arising from more distal synapses ${ }^{84-86}$. To examine whether the CNN-LSTM took distal inputs into consideration or neglected these inputs in favor of more robust proximal ones, we inspected the weights of the first layer of the neural net architecture (Figure 3B). This convolutional layer consists of 512 filters, which directly processes the input matrix (64 ms of 201 input vectors corresponding to the somatic membrane potential and vectorized timing information of 200 synapses). Despite the random initialization of these filters from 
bioRxiv preprint doi: https://doi.org/10.1101/2021.02.22.432356; this version posted February 23, 2021. The copyright holder for this preprint (which was not certified by peer review) is the author/funder, who has granted bioRxiv a license to display the preprint in perpetuity. It is made available under aCC-BY-NC-ND 4.0 International license.

A
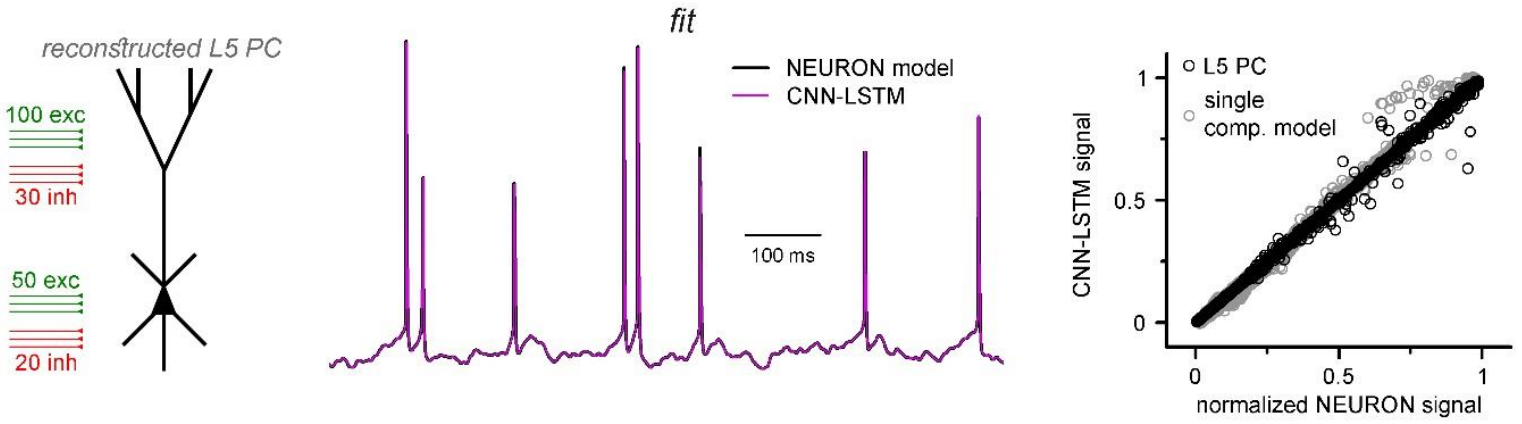

B

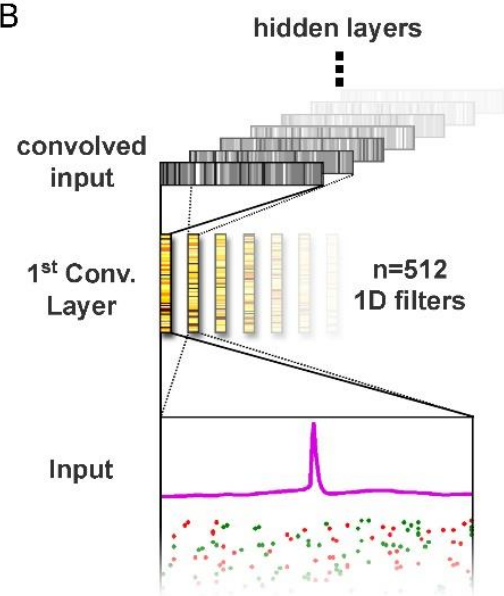

C

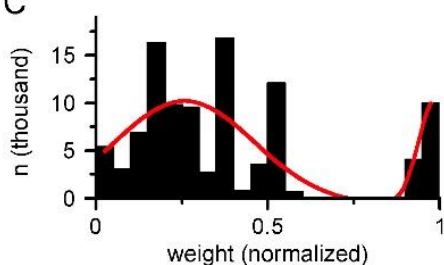

D

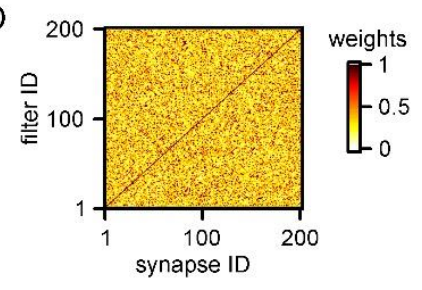

E

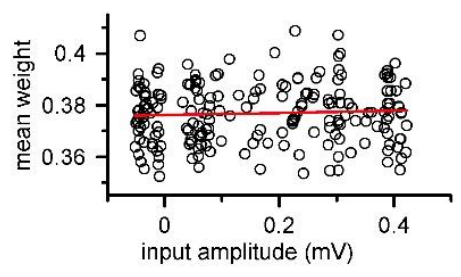

$\mathrm{F}$

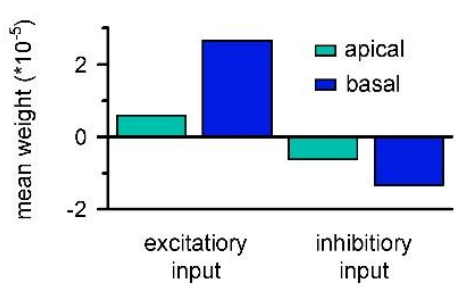

G prediction

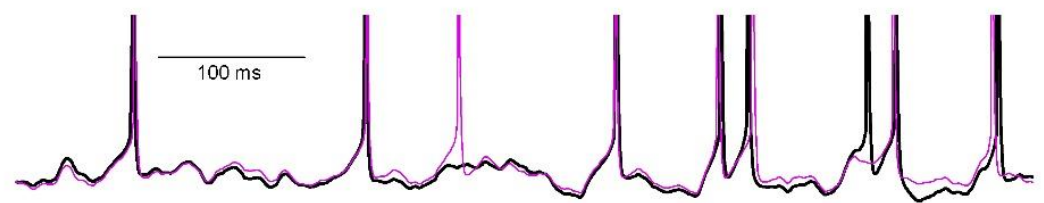

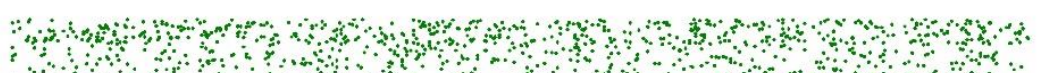

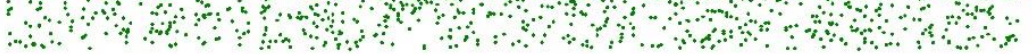

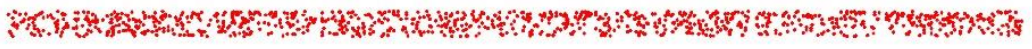

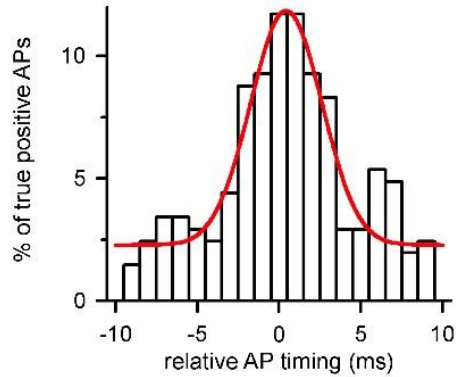

Figure 3. Multicompartmental simulation representation by CNN-LSTM. A. CNN-LSTM can accurately predict membrane potential of a multicompartmental neuron upon distributed synaptic stimulation. Representative figure depicts the placement of synaptic inputs onto apical and basal dendrites of a reconstructed L5 PC (left). Point-by-point forecasting of L5 PC membrane potential by a CNN-LSTM superimposed on biophysically detailed NEURON simulation (left). CNN-LSTM prediction accuracy of multicompartmental membrane dynamics is comparable to single compartmental simulations (right, L5 PC in black, single compartmental simulation of Figure $1 \mathrm{D}$ in grey, $n=45000$ and 50000 respectively). B. Convolutional filter information was gathered from the first convolutional layer (middle, color scale depicts the different weights of the filter), which directly processes the input (membrane potential in magenta, excitatory and inhibitory synapse onsets in green and red respectively), provind convolved inputs to upper layers (grey bars, showing the transformed 1D outputs). C. Distribution of filter weights from 512 convolutional units $(n=102400)$ with double Gaussian fit (red). E. Filter weight is independent of the somatic amplitude of the input (circles are averages from 512 filters, $n=200$, linear fit in red). D. Each synapse has a dedicated convolutional unit, shown by plotting the filter weights of the 200 most specific units against 200 synapses. Notice the dark diagonal illustrating high filter weights. F. Excitatory and inhibitory snypase information is convolved by fitlers with opposing weights ( $n=51200$, 25600, 15360 and 10240 for apical excitatory, basal excitatory, apical inhibitory and basal inhibitory synapses respectively). G. Representative continous prediction of L5 PC membrane dynamics by CNN-LSTM (magenta) compared to NEURON simulation (black) upon synaptic stimulation (left, excitatory input in green, inhibitory input in red). Spike timing is measured on subthreshold traces (right, $\mathrm{n}=50$ for variance explained, precision and recall).

a uniform distribution ${ }^{87}$, only a small fraction of optimized filter weights were selected for robust 
information representation (13.83\% of all weights were larger than 0.85$)$, while the majority of them were closer to zero (Figure 3C) suggesting relevant feature selection. In order to demonstrate that this feature selection was not biased against distal inputs, the 512 convolutional filters were ranked by their selectivity for distinct synapses. We found that each synaptic input was assigned an independent selectivity filter (Figure 3D). Next, we compared the mean weights of each synapse with the somatic amplitude of the elicited voltage response (Figure 3E). This comparison revealed a flat linear correspondence (Pearson's $r=0.06$ ), which combined with the filter specificity (Figure 3D) confirmed that distal and proximal synaptic inputs carry equally relevant information for the CNN-LSTM.

Interestingly, when we compared the weights of excitatory and inhibitory inputs, we found that even at the first layer, the CNN-LSTM is able to determine that these inputs have opposing effects on subsequent membrane potential $\left(5.91 * 10^{-6}, 2.66 * 10^{-5},-6.22 * 10^{-6}\right.$ and $-1.34 * 10^{-5}$ mean weights for apical excitatory, basal excitatory, apical inhibitory and basal inhibitory synapses respectively, $\mathrm{n}=$ 51200, 25600, 15360 and 10240) even though these vectors only contain synaptic conductance information (comparable positive values for both excitatory and inhibitory synapses, Figure 3F). Taken together, the feature selectivity and prediction accuracy confirm that the CNN-LSTM architecture is well suited for representing multicompartmental information.

\section{Ultra-rapid single neuron and network simulations using CNN-LSTM}

Although our results demonstrate that ANNs are able to replicate major features of neuronal behavior such as subthreshold and spiking dynamics (Figure 1), adequately generalize (Figure 2), and accurately represent multicompartmental information (Figure 3), one of the main reasons why we chose a machine learning approach as a substitute for traditional modeling environments is the potential for markedly reduced simulation runtimes. Simulation environments such as NEURON rely on compartment specific mathematical abstractions of active and passive biophysical mechanisms ${ }^{63}$, which results in high computational load in increasingly complex neuronal models. Furthermore, the number of equations to be solved linearly increases with the number of simulated cells in a circuit. Although several attempts have been made to reduce the demanding computational load of neuronal simulations $^{32,33,88-92}$, the most widely used approach is parallelization, both at the level of single cells ${ }^{93}$ and network models ${ }^{94,95}$. In case of moderately sized ${ }^{96-100}$ and full-scale networks ${ }^{19-21,101}$ this hinders the possibility of running these models on widely available computational resources, instead users and developers are forced to employ supercomputers. However, ANNs offer a possible solution to this problem. Contrary to traditional modeling environments, the graph-based ANNs created by the machine learning platform we choose are specifically designed for parallel information processing. 
A

SIMULATING

SINGLE CELL

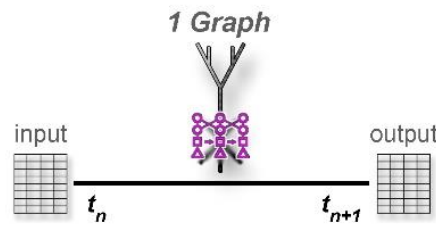

1 set of equations

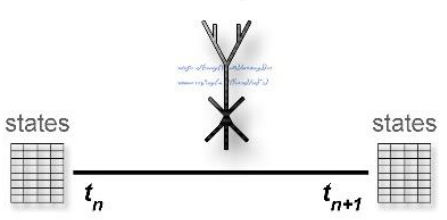

SIMULATING

MULTIPLE CELLS

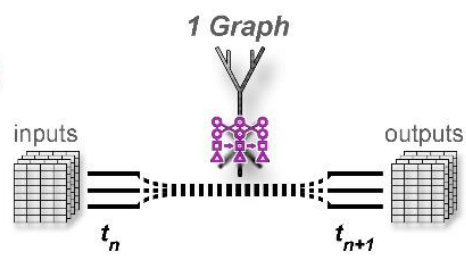

$n$ sets of equations

NEURON

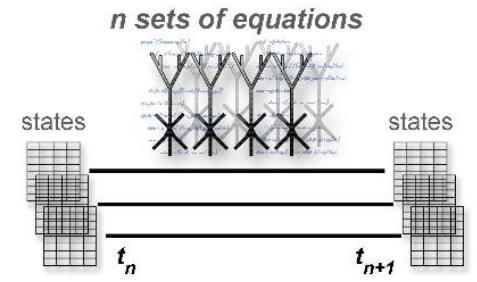

B

Simulation of simplified point neuron

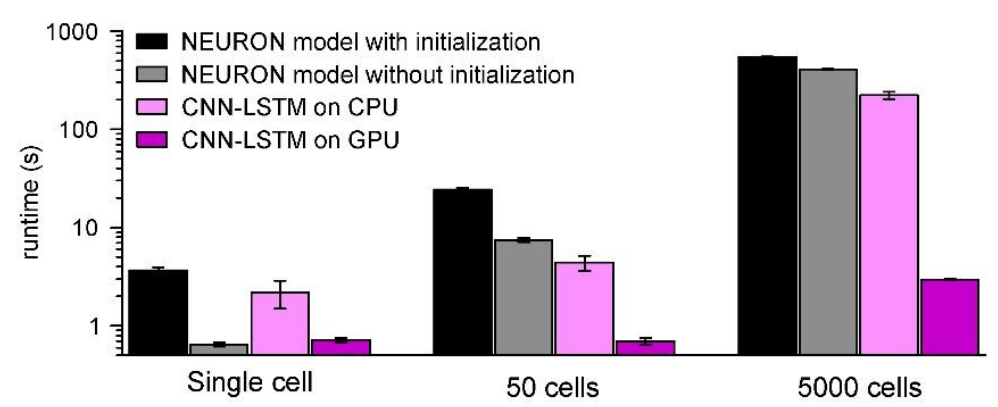

C

Simulation of detailed L5 pyramidal cell

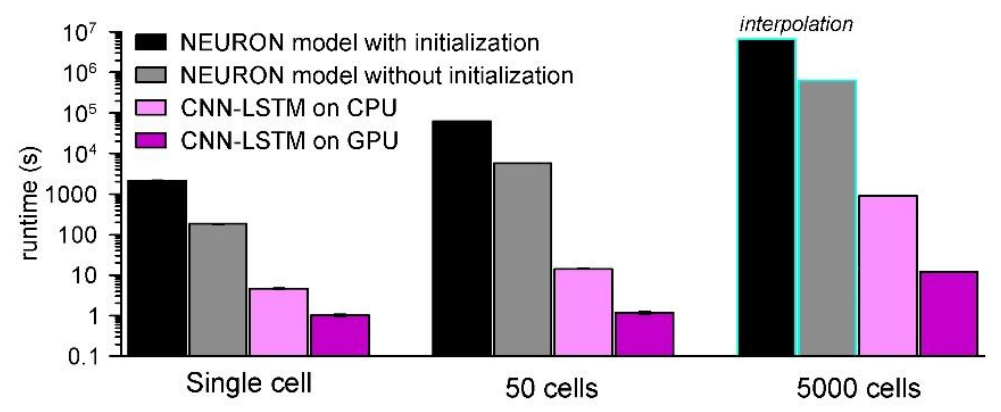

Figure 4. Orders of magnitude faster simulation times with CNN-LSTM. A. An illustration demonstrating that CNN-LSTMs (top, magenta) handle both single cell (left) and network (right) simulations with a single graph, while the set of equations to solve increases linearly for NEURON simulations (bottom, black). B. 100 ms simulation runtimes of a one, 50 and 5000 point neurons on four different resources. Bar graphs represent the average of five sumilations. C. Same as in panel B, but for L5 PC simulations. Teal borders represent interpolated datapoints.

This means that ANN simulation run times do not increase linearly by the inclusion of additional cells into the simulated circuit (Figure 4A), resulting in better scaling for large networks where an immense number of cells of the same cell type are simulated.

To verify the efficiency of our CNNLSTM, we compared single cells and moderate- to largescale network simulation

runtimes against NEURON models used in Figure 1 and Figure 3. NEURON simulations were performed on a single central processing unit (CPU), as this is the preferred and most widely used method (although see ${ }^{102}$, while neural nets were run on both CPU and graphical processing unit (GPU) because these calculations are optimized for GPUs, in which much larger number of processing units are available. We found that in case of point neurons, when the optional initialization step was omitted, single cell simulations ran significantly faster in NEURON than their CNN-LSTM counterparts (Figure 4B, $3.68 \pm 0.24 \mathrm{~s}, 0.65 \pm 0.03 \mathrm{~s}, 2.19 \pm 0.69 \mathrm{~ms}$ and $0.72 \pm 0.04 \mathrm{~s}, 100 \mathrm{~ms}$ cellular activity by NEURON with initialization, NEURON without initialization, CNN-LSTM on CPU and CNN-LSTM on GPU 
respectively, $n=5$ ). However, with increased network size, the predicted optimal scaling of CNNLSTM models resulted in faster runtimes compared to NEURON models for a 50-cell network (24.23 $\pm 1.12 \mathrm{~s}, 7.45 \pm 0.37 \mathrm{~s}, 4.42 \pm 0.77 \mathrm{~s}$ and $0.71 \pm 0.05 \mathrm{~s}$ for simulating $100 \mathrm{~ms}$ network activity by NEURON with initialization, NEURON without initialization, CNN-LSTM on CPU and CNN-LSTM on GPU respectively, $n=5$ ). These results show that while in NEURON the runtimes increased by approximately 6.6-times, CNN-LSTM runtimes on a GPU did not increase.

In order to demonstrate the practicality of ANNs for typical large-scale network simulations, we repeated these experiments with 5000 cells (representing the number of cells in a large-scale network belonging to the same cell type ${ }^{21}$ ). In these conditions, the NEURON simulation was $\sim 148$ times slower than a single cell simulation. Importantly, the large-scale CNN-LSTM simulation only slowed by a factor of 4 with respect to a single cell (Figure 4B, $546.85 \pm 4.61 \mathrm{~ms}, 407.2 \pm 9 \mathrm{~ms}$, $222.15458 \pm 19.02 \mathrm{~ms}$ and $2.97 \pm 0.02 \mathrm{~ms}$ for simulating $100 \mathrm{~ms}$ network activity by NEURON with initialization, NEURON without initialization, CNN-LSTM on CPU and CNN-LSTM on GPU respectively, $\mathrm{n}=5$ ).

We next compared runtime disparities for NEURON and CNN-LSTM simulations of detailed biophysical models. Interestingly, we found that the single cell simulation of the L5 PC model ran significantly slower than the CNN-LSTM abstraction $\left(2.08 * 10^{3} \pm 84.66 \mathrm{~s}, 185.5 \pm 3.7 \mathrm{~s}, 4.73 \pm 0.13 \mathrm{~s}\right.$ and $1.02 \pm 0.05 \mathrm{~s}$ for simulating $100 \mathrm{~ms}$ network activity by NEURON with initialization, NEURON without initialization, CNN-LSTM on CPU and CNN-LSTM on GPU respectively, $n=5$ ). The runtime disparity was markedly amplified in network simulations (50 cell network: $6.3 * 10^{4} \mathrm{~s}, 5.8 * 10^{3} \mathrm{~s}, 14.3$ $\pm 0.24 \mathrm{~s}$ and $1.19 \pm 0.08 \mathrm{~s}, 5000$ cell network: $6.53 * 10^{6} \mathrm{~s}, 6.28 * 10^{5} \mathrm{~s}, 901.15 \mathrm{~s}$ and $11.99 \mathrm{~s}$ for simulating 100 ms network activity by NEURON with initialization, NEURON without initialization, CNN-LSTM on CPU and CNN-LSTM on GPU respectively, $n=5$ ), resulting in a four to five orders of magnitude faster runtime (depending on initialization) for the CNN-LSTM in case of large-scale network simulations. These results demonstrate that our machine learning approach yields far superior runtimes compared to traditional simulating environments. Furthermore, the introduced speed-up is comparable to the number of parallel CPU cores used for several network simulations ${ }^{19-21}$ introducing the possibility of running large or full-scale network simulations on what are now widely available computational resources.

\section{Parameter space optimization using ANNs}

Many important advancements have recently been made in the development of detailed, largescale network simulations, however, the bottleneck continues to be slow simulation runtimes. Consequently, network simulations can be carried out only a few times (but see ${ }^{103}$ ), hindering 
imperative network construction steps, such as parameter space optimization. To establish whether this bottleneck could be remedied by the drastic runtime acceleration with ANNs, we explored the influence of several network parameters on circuit behavior in a model of Rett syndrome. This neurodevelopmental disorder is caused by loss-of-function mutations in the X-linked methyl-CpG binding protein $(\mathrm{MeCP} 2)^{45}$. Rett syndrome occurs in $\sim 1: 10,000$ births worldwide, resulting in intellectual disability, dysmorphisms, declining cortical and motor function, stereotypies, and frequent myoclonic seizures mostly in girls ${ }^{104-110}$. Although the underlying cellular and network mechanisms are largely unknown, changes in synaptic transmission ${ }^{111-113}$, morphological alterations in neurons ${ }^{107}$, ${ }^{114}$ and altered network connectivity ${ }^{115}$ have been reported in Rett.

We aimed to investigate the contribution of distinct alterations on cortical circuit activity in Rett using a recurrent L5 PC network ${ }^{98}$ composed entirely of CNN-LSTM-L5-PCs (Figure 5A). Simulations were run uninterrupted for $100 \mathrm{~ms}$, when a brief $(1 \mathrm{~ms})$ perisomatic excitation was delivered to mimic thalamocortical input onto thick tufted pyramidal cells ${ }^{116-118}$. In control conditions, cells fired well-timed APs rapidly after the initial stimuli (Figure 5B). Following this immediate response, extended AP firing can occur as a consequence of the circuit recurrent connectivity. (Figure $5 \mathrm{~B},{ }^{119,}{ }^{120}$ ). In network conditions corresponding to Rett syndrome (reduced recurrent connectivity between L5 PCs, reduced excitatory and inhibitory drive onto L5 PCs), we observed the emergence of circuit-wide oscillations (Figure 5C). Cortical networks endowed with frequent recurrent connections between excitatory principal cell are prone to exhibit oscillatory behavior, which is often the mechanistic basis of pathophysiological network activities ${ }^{121}$. Therefore, we quantified oscillatory activity $^{122-124}$ and the immediate response to thalamocortical stimuli independently (Figure 5C). By systematically changing inhibitory quantal $\operatorname{size}^{125}$ and the ratio of recurrent L5 PC innervation ${ }^{115}$, we found that recurrent connectivity is the major determinant of network instability, as connectivity below 9\% never resulted in oscillatory activity (Supplementary Figure 3, $9.14 \pm 2.21$ vs $320.78 \pm 237.66$ APs, $\mathrm{n}=1740$ vs 760 for below $9 \%$ connectivity and connectivity between $9-15 \%$ respectively, $\mathrm{p}=2.2^{*} 10^{-}$ ${ }^{219}$, two sample t-test). Interestingly, we found no measurable relationship between the inhibitory quantal size and the network response to thalamocortical stimuli either (133.62 \pm 29.32 vs $131.72 \pm$ 32.32 APs upon thalamocortical stimulus for inhibitory input scaling of 1 and 0.5 , respectively, $\mathrm{n}=50$ 
each, $\mathrm{p}=0.76, \mathrm{t}(98)=0.31$, Two Sample t-Test), which suggests that these metrics are robust for perturbations of the inhibitory circuitry in baseline conditions. Therefore, our next aim was to investigate the effect of different levels of excitation on the network. Contrary to disruption of the inhibitory system, we found that both changes in the excitatory drive and recurrent connectivity had considerable influence over network instability (Figure 5D, excitatory drive: $17.85 \pm 61.61$ vs 388.92 \pm 170.03 pre-stimulus APs for excitatory drive scaled by 0.75 and 1.25 , respectively, $\mathrm{n}=100$ each, $\mathrm{p}$ $=6.73 * 10^{-51}, \mathrm{t}(198)=-20.52$, Two Sample t-Test; recurrent connectivity: $321.96 \pm 200.42$ vs $157.66 \pm$ 192.5 pre-stimulus APs for $10 \%$ and $5.2 \%$ recurrent connectivity, similar to reported values for adult

A

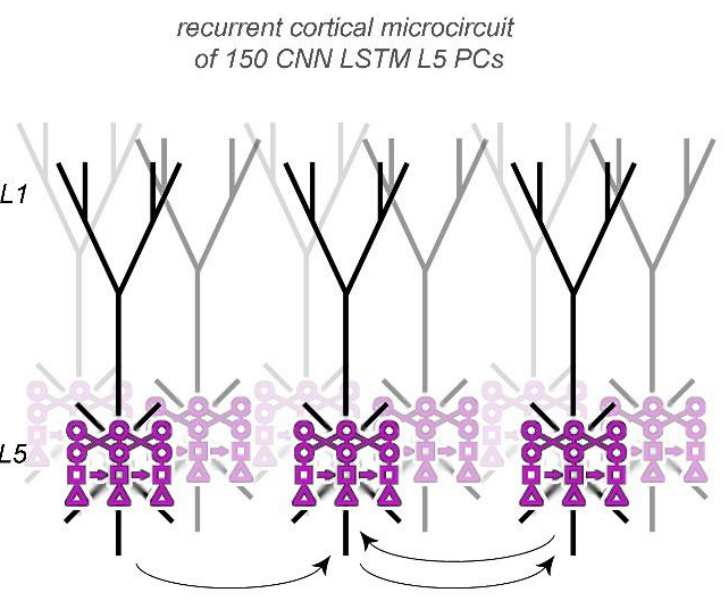

C

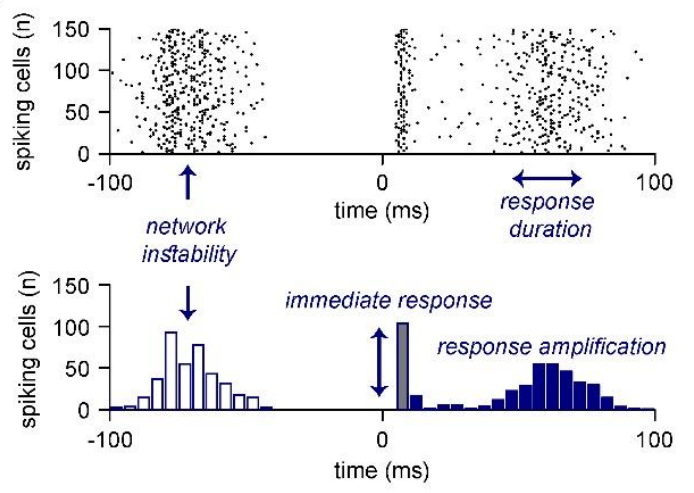

E

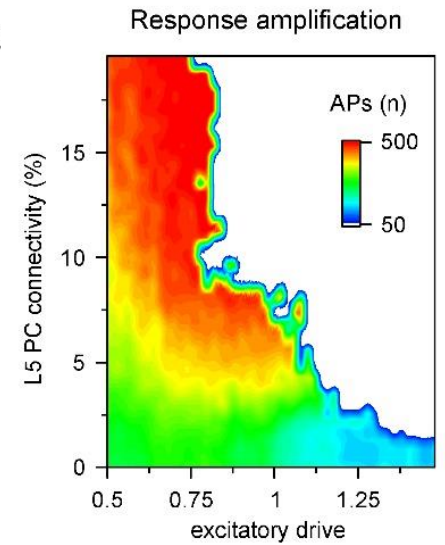

Response duration

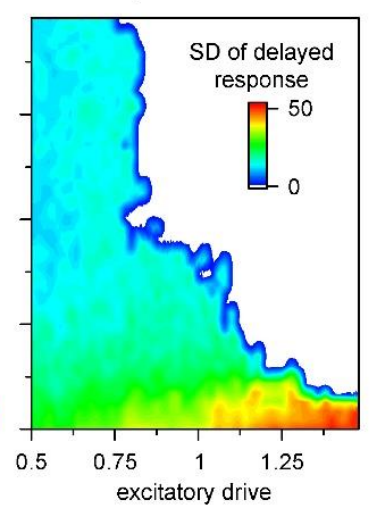

B
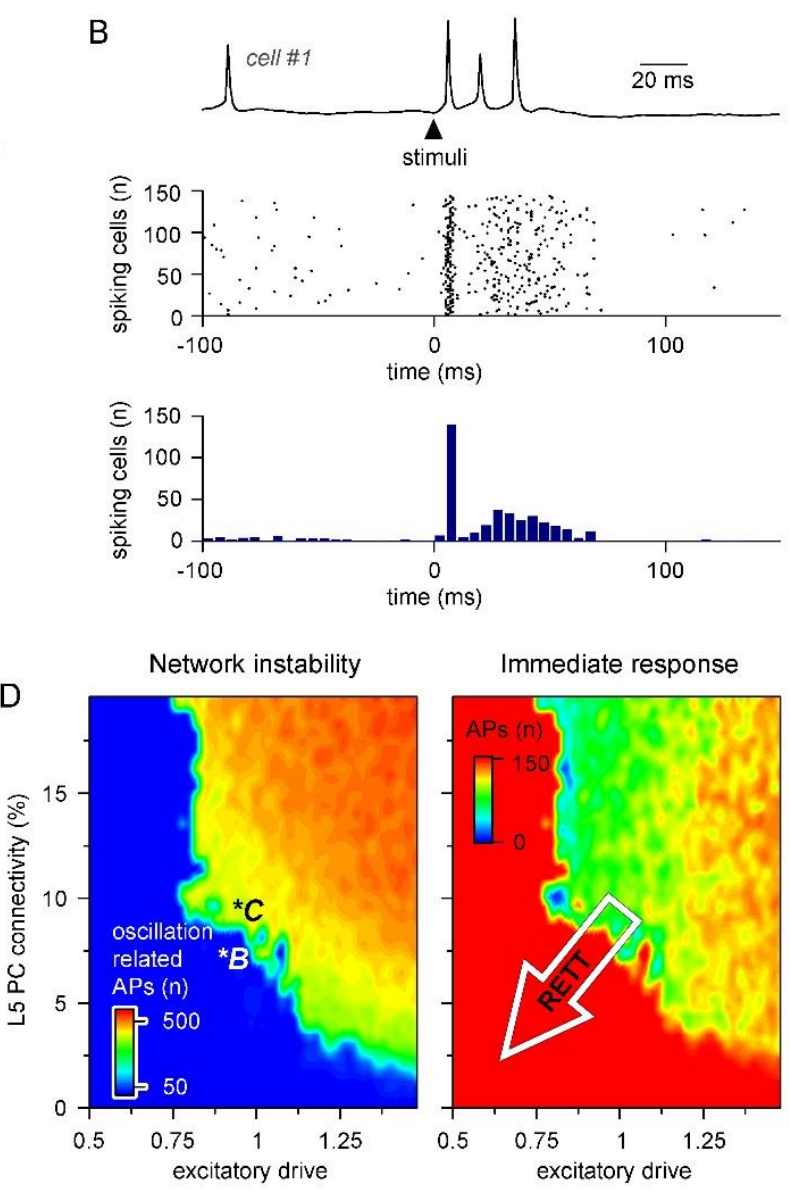

F

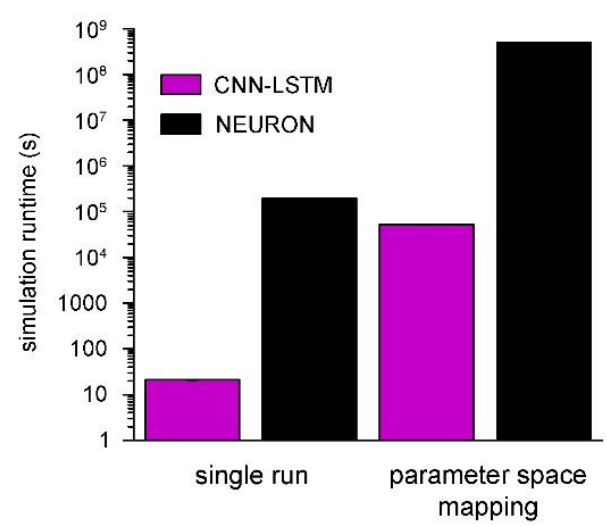


Figure 5. Efficient parameter-space mapping with CNN-LSTMs reveals a joint effect of reccurrent connectivity and E/I balance on network stability and efficacy in Rett syndrome. A. 150 CNN-LSTM models of L5 PCs were simulated in a recurrent microcircuit . B. The experimental setup consisted of a stable baseline condition for $100 \mathrm{~ms}$, a thalamocortical input at $\mathrm{t}=100$ $\mathrm{ms}$, and network recponse, monitored for $150 \mathrm{~ms}$. Example trace from the first simulated CNN-LSTM L5 PC on top, raster plot of $150 \mathrm{~L} 5 \mathrm{PCs}$ in the middle, number of firing cells with $5 \mathrm{~ms}$ binning for the same raster plot in the bottom. Time is alligned to the stimulus onset ( $t=0$, black arrowhead). C. Four parameters were quantified; network instability (number of cells firing before the stimulus), immediate response (number of cells firing within $10 \mathrm{~ms}$ of the stimulus onset), reponse duration (SD of the spikes following the stimulus) and response amplification (number of cells firing after stimulus onset). The example simulation depicts highly unstable network conditions. D. Netwrok instability (left) and immediate response (right) as a function of altered L5 PC connectivity and excitatory drive. ${ }^{*} \mathrm{~B}$ and ${ }^{*} \mathrm{C}$ indicated network parameters used for generating panel $\mathrm{B}$ and panel C, respectively. E. Response amplification (left) and reponse duration (right) as a function of L5 PC recurrent connectivity and excitatory drive. Only stable network conditions were quantified as instability contaminates these measurements. F. Simulation runtime for single simulation (left, network of 150 cells simulated for $250 \mathrm{~ms}$ ) and parameter space mapping (right, 150 cells simulated for 250 ms, 2500 times, for generating panel D and F).

wild type and Mecp2-null mutant mice ${ }^{115}, \mathrm{n}=50$ each, $\mathrm{p}=6.33^{*} 10^{-5}, \mathrm{t}(98)=-4.18$, Two Sample $t$ Test) and response to stimuli (excitatory drive: $147.58 \pm 17.2$ vs $119.23 \pm 18.1$ APs upon stimulus for excitatory drive scaled by 0.75 and 1.25 , respectively, $\mathrm{n}=100$ each, $\mathrm{p}=2.3 * 10^{-22}, \mathrm{t}(198)=11.03$, Two Sample t-Test; recurrent connectivity: $134.76 \pm 21.37$ vs $112.74 \pm 34.99$ APs upon stimulus for $10 \%$ and $5.2 \%$ recurrent connectivity, $\mathrm{n}=50$ each, $\mathrm{p}=2.54 * 10^{-4}, \mathrm{t}(98)=3.8$, Two Sample $t$-Test . Next, we quantified the ability of the recurrent network to amplify thalamocortical inputs. In stable network conditions, recurrent connectivity and the level of excitation had similar effects on the strength (Figure 5E, excitatory drive: $436.74 \pm 72.38$ vs $160.64 \pm 16.31, \mathrm{n}=98$ and 14 post-stimulus APs for excitatory drive scaled by 0.75 and 1.25 , respectively, $\mathrm{p}=1.46^{*} 10^{-26}, \mathrm{t}(110)=14.17$, Two Sample $t$ Test; recurrent connectivity: $441.14 \pm 32.7$ vs $392.73 \pm 26.81, \mathrm{n}=30$ and 14 pre-stimulus APs for $10 \%$ and $5.2 \%$ recurrent connectivity, respectively, $\mathrm{p}=5.55^{*} 10^{-6}, \mathrm{t}(42)=-5.2$, Two Sample t-Test) and duration of network responses (excitatory drive: $16.75 \pm 4.95$ vs $39.05 \pm 5.52, \mathrm{n}=98$ and 14 network response duration for excitatory drive scaled by 0.75 and 1.25 , respectively, $\mathrm{p}=1.51 * 10^{-29}, \mathrm{t}(110)=$ 15.56, Two Sample t-Test; recurrent connectivity: $16.13 \pm 2.23$ vs $13.44 \pm 1.82, \mathrm{n}=30$ and 14 prestimulus APs for $10 \%$ and $5.2 \%$ recurrent connectivity, respectively, $\mathrm{p}=2.01 * 10^{-4}, \mathrm{t}(42)=-4.07$, Two Sample t-Test-). These results suggest that lowered recurrent connectivity reduces network instability. Specifically, recurrent connectivity observed in young Mecp2-null mice $\left(7.8 \%{ }^{115}\right)$ yielded more stable microcircuits ( $54 \%$ of networks were stable, $\mathrm{n}=100$ ), than wild-type conditions (34\% of networks were stable, $n=50)$. Recurrent connection probability of older animals $(5.3 \%)$ further stabilized this network (64\% of networks were stable). Taken together, our model suggests that reduced recurrent connectivity between L5 PCs is not causal to seizure generation and abnormal network activity ${ }^{110,122}$, which are crucial symptoms of Rett syndrome at young age, instead it actively suppresses structured pyramidal cell activation. 
Finally, we compared the run time of the simulated layer 5 microcircuit of NEURON and CNNLSTM models. We found that for a single simulation, CNN-LSTM models were more than 9300-times faster compared to NEURON models (Figure $5 \mathrm{f}, 21.153 \pm 0.26 \mathrm{~s}$ vs $54.69 \mathrm{~h}$ for CNN-LSTM and NEURON models respectively). This unparalleled acceleration for multicompartmental neuronal information processing demonstrates that parameter space optimization is not only attainable by CNNLSTM models on commercially available computational resources, but it is almost 4-times faster than completing a single NEURON simulation.
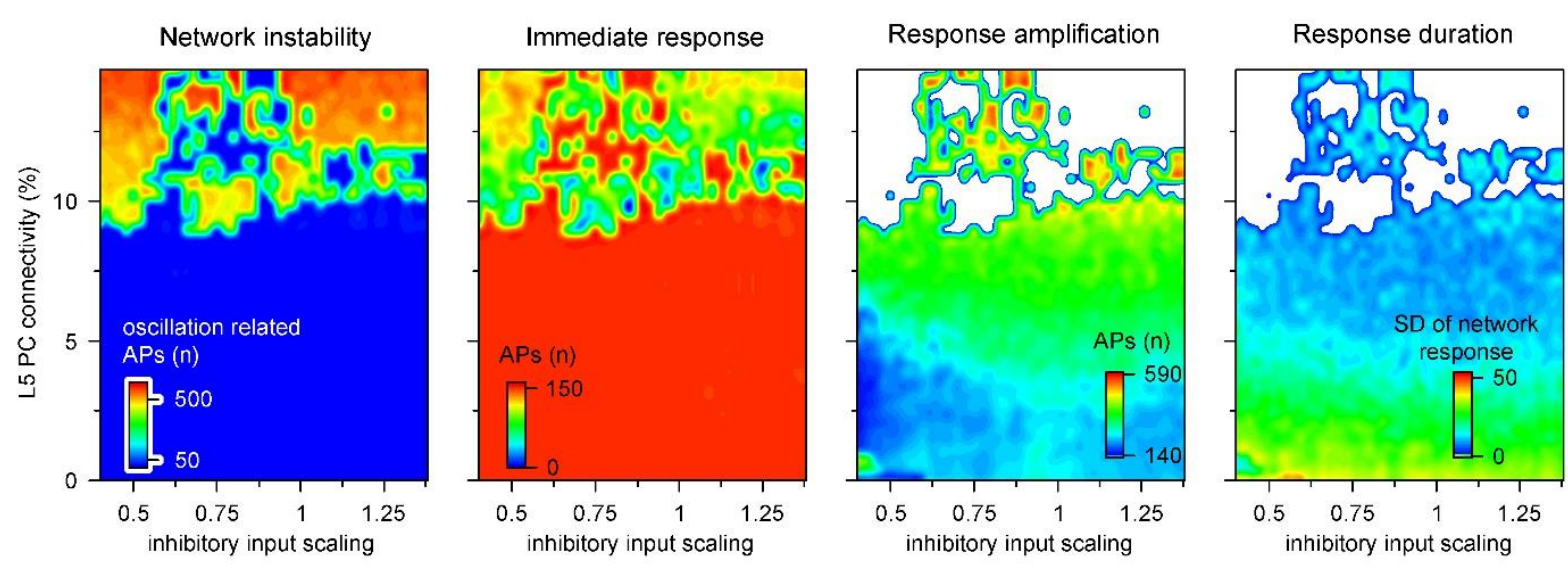

Supplementary Figure 3. Microcircuit stability and efficacy is robust to changes in inhibitory drive. Network parameters were quantified as shown in Figure 5 panel C. For reponse amplification and response duration only stable network conditions are shown (right).

\section{DISCUSSION}

In this study, we present an ANN architecture (CNN-LSTM) capable of accurately capturing neuronal membrane dynamics. Most of the investigated ANN architectures predicted subthreshold voltage fluctuations of point-neurons, however only the CNN-LSTM was able to generate action potentials. This model was able to generalize well to novel input and could also predict various other features of neuronal cells, such as voltage-dependent ionic current dynamics. Furthermore, the CNN-LSTM accounted for the majority of the variance of subthreshold voltage fluctuations of biophysically realistic L5 PC models with excitatory and inhibitory synapses distributed along the entirety of the dendritic tree. The timing of the predicted action potentials closely matched the ground truth data. Importantly, we found that although single cell simulation runtimes are comparable, the CNN-LSTM have superior scaling for large network simulations. Specifically, in case of mid-sized biophysically detailed networks (50 cells), ANNs were more than three orders 
of magnitude faster, while for large-scale networks (5000 cells) ANNs are predicted to be five orders of magnitude faster than traditional modeling systems. These accelerated simulation runtimes allowed us to easily investigate a L5 PC network in distinct conditions, for example, to uncover network effects of altered connectivity and synaptic signaling observed in Rett syndrome. In our Rett cortical circuit model, recurrent connectivity and excitatory drive jointly shape network stability and responses to sensory stimuli, showing the power of this approach in generating testable hypotheses for further empirical work. Together, the described model architecture provides a suitable alternative to traditional modeling environments with superior simulation speed for biophysically detailed cellular network simulations.

\section{ANNs can accurately predict the full range of neuronal membrane potential dynamics}

As our familiarity with neuronal circuits grows, so does the complexity of models tasked with describing their activity. Consequently, supercomputers are a regular occurrence in research articles which describe large-scale network dynamics built upon morphologically and biophysically detailed neuronal models ${ }^{19-21}$. Here we developed an alternative to these traditional models, which can accurately represent the full dynamic range of neuronal membrane voltages in multicompartmental cells, but with substantially accelerated simulation runtimes.

ANNs are ideal substitutes to traditional model systems for several reasons. First, ANNs do not require hard-coding of the governing rules for neuronal signal processing. When an ANN is created, it serves as a blank canvas which can derive the main principles of input-output processing and neglect otherwise unimpactful processes ${ }^{126-128}$. The degree of simplification depends only on the ANN itself, not the developer, thereby reducing human-errors. However, architecture construction and training dataset availability represent limiting steps in ANN development ${ }^{54}$. Fortunately, the latter issue is void as virtually infinite neuronal activity training datasets are now available for deep learning. On the other hand, as we have demonstrated, the former concern can significantly impede ANN construction. Although we have shown that subthreshold signal processing can be accurately depicted by markedly divergent ANN architectures, we found only one that was suitable for both subthreshold active membrane potential prediction. This does not mean that the presented architecture is the only possible ANN model for neural simulations, as the machine learning is a rapidly progressing field that frequently generates highly divergent ANN constructs ${ }^{129}$. The importance of the network architecture is further 
emphasized by our findings demonstrating that ANNs with comparable or even greater numbers of freely adjustable parameters were unable to handle suprathreshold information.

The prevailing CNN-LSTM architecture was proven to be suitable for depicting membrane potential and ionic current dynamics of both simplified and biophysically detailed neuronal models and generalized well for previously unobserved simulation conditions. These results therefore prove that ANNs are ideal substitutes for traditional model systems for representing various features of neuronal information processing in a significantly accelerated simulations. Future architecture alterations should focus on continued improvement of action potential timing and prediction.

\section{ANN simulations drastically reduce the run time for large-scale network simulations}

Accelerated simulation runtimes are particularly advantageous for large-scale biological network simulations, which have seen an unprecedented surge in recent years. These network simulations not only provide support for experimentally gathered information, but also as testing benchmarks in the future for several network related queries such as pharmaceutical target testing and for systemic interrogation of cellular level abnormalities in pathophysiological conditions ${ }^{130-136}$. However, widespread adaptation of large-scale network simulations is hindered by the computational demand of these models that can only be satisfied by the employment of supercomputer clusters ${ }^{19-21}$. Because these resources are expensive, they do not constitute a justifiable option for general practice. Importantly, we have shown that ANNs can not only provide a suitable alternative to traditional modeling systems, but that their simulation runtimes are superior to them as well, due to the structure of the machine learning platform, in this case Tensorflow.

Traditional model systems linearly increase the number of equations to be solved for parallelly simulated cells, while ANNs can handle cells belonging to the same cell type on the same ANN graph ${ }^{137}$. In our network models (150 cells; Figure 5), NEURON simulations yield 150-times higher number of linear equations every time step, while ANNs used the same graph for all simulated cells. This property of ANNs in particular suits biological networks consisting of many cells of the same type, such as the ones being developed in recent years. For example, the Allen Institute recently published a computational model of the mouse V1 cortical area ${ }^{21}$, consisting of 17 different cell types (with the number of cells corresponding to these cell types 
range from hundreds to more than ten thousands), which means that a complete cortical area can be simulated using only 17 ANNs. We have demonstrated that even for small networks consisting of only 150 cells of the same type, ANNs are more than four orders of magnitude faster compared to model environments used in the aforementioned V1 simulations. As large-scale network simulations are typically run using several thousand CPU cores in parallel, the provided run time acceleration suggests that network simulations relying on ANNs could negate the need for supercomputers. Instead, these models can be run on commercially available computational resources such as personal computers with reasonable timeframes.

Another advantage of our approach is the utilization of GPU processing, which provides substantially larger number of processing $\operatorname{cores}^{138,139}$. The runtime difference that can be attributed to the employed computational resource can be seen by comparing CNN-LSTM simulations on CPU and GPU (Figure 4 b, c), which yields below an order of magnitude faster simulations on GPU in the case of moderate size networks (50 cells) and approximately two orders of magnitude difference for large networks. However, our results demonstrated that cortical pyramidal cell networks simulations are at least four orders of magnitude faster than traditional modeling environments, which confirms that the disparity in the number of cores can only partially account for the observed runtime acceleration. Furthermore, the NEURON simulation environment does not benefit as much from GPU processing as ANN simulations ${ }^{102,140}$. These results confirm that the drastic runtime acceleration is the direct consequence of the parallelized graph-based ANN approach.

\section{ANNs can efficiently predict changes in network function in complex brain disorders}

To demonstrate the superiority of ANNs in a biologically relevant network simulation, we mapped the effects of variable network parameters observed in Rett syndrome. Rett syndrome is a neurodevelopmental disorder leading to a loss of cognitive and motor functions, impaired social interactions, and seizures in young females due to loss of function mutations in the X-linked $M e C P 2$ gene $^{45}$ ). Like many brain diseases, these behavioral alterations are likely due to changes in several different synaptic and circuit parameters. MeCP2-deficient mice exhibit multiple changes in synaptic communication, affecting both excitatory and inhibitory neurotransmission and circuit-level connectivity as well. Excitatory transmission is bidirectionally modulated by MeCP2 knock-out ${ }^{141,142}$ and overexpression ${ }^{143}$, and long term synaptic plasticity is also impaired 
in MeCP2-deficient mice ${ }^{144,145}$. Inhibitory signaling is also altered in several different brain $\operatorname{areas}^{111,112}$. Importantly, synaptic transmission is not only affected at the level of quantal parameters, but at the number of synaptic connections, because MeCP2 directly regulates the number of glutamatergic synapses ${ }^{142}$. This regulation amounts to a $39 \%$ reduction of putative excitatory synapses in the hippocampus ${ }^{142}$, and a $50 \%$ reduction in recurrent excitatory connections between layer 5 pyramidal cells ${ }^{115}$. Here we investigated how these diverse underlying mechanisms contribute to overall circuit pathology using our ANN network model approach.

We found that the ability of the network to respond to external stimuli is affected by both alterations in synaptic excitation and changes in the recurrent connectivity of layer 5 pyramidal cells. Our results suggest that disruption of inhibitory transmission is not necessary to elicit network instability in Rett, as changes in synaptic excitation and recurrent connectivity alone were sufficient in destabilizing the network. These results are supported by previous findings showing that both constitutive ${ }^{146}$ and excitatory cell targeted ${ }^{147} M e C P 2$ mutation leads to network seizure generation, as opposed to inhibitory cell targeted $M e C P 2$ mutation, which causes frequent hyperexcitability discharges but never seizures ${ }^{125}$. Furthermore, our results suggest that excitatory synaptic alterations in Rett affect both general network responses and network stability, which may serve as substrates to cognitive dysfunction and seizures, respectively. Taken together, these results revel how cellular-synaptic mechanisms may relate to symptoms at the behavioral level. Importantly, investigation of the multidimensional parameter space was made possible by the significantly reduced simulation times of our ANN, as identical simulations with traditional modelling system are proposed to be four orders of magnitude slower.

\section{Future directions}

In this study, we present an ANN architecture suitable for investigation of membrane dynamics in neural cells in a drastically accelerated time frame. The proposed modeling solution has the promise to not only expedite large scale network simulations, but to achieve hyperparameterspace optimization in these networks as well, which was previously unattainable. Importantly, optimization of the Python code was kept to the minimum because our aim was to demonstrate the advantages of ANN over traditional simulation environments. Therefore, the reported simulation runtimes can be considered moderately overestimated. As a considerable portion of numerical calculations is spent on solving equations related to synaptic plasticity and 
establishing connections, it would greatly benefit the simulation timeframe if this part of the Python code would be optimized. This can be done in at least two separate, but non-exclusive ways. First, numerical operations of Python can be optimized by employing language extensions (such as Cython ${ }^{148}$ or Numba ${ }^{149}$ ), which allows code compiling to faster languages. Second, we deliberately did not take advantage of specific parallelization options offered by Python. Currently, commercially available processors can offer 6 to 8 cores, which with optimum thread-count can run 12-15 processes in parallel. Interestingly, this number closely resembles the number of cell types typically represented in circuit models. Therefore, entire circuit models with could be efficiently parallelized using different neuron types with distinct biophysical properties in the near future.

In contrast to traditional modeling systems, the bottleneck for network simulations with multiple neuron types will not be the simulation runtime itself, but the generation of accurate ANN descriptions for each distinct neuron. This may be expedited by transfer learning ${ }^{150,151}$. Transfer learning uses previously acquired knowledge of trained neural networks to solve new, but similar problems more quickly. In other words, if an ANN can learn basic signal processing mechanisms on neural cells, it can be retrained to represent neural dynamics of a morphologically similar neuron. For example, interneurons with similar branching or excitatory cells with similar topologies can be expeditiously cross-trained.

In conclusion, we described a machine learning framework for simulating membrane potential dynamics of complex neural cells in a substantially accelerated timeframe. Our ANN approach allows for efficient large-scale network simulations without compromising biologically relevant cellular features, such as multicompartmental information processing. We propose that ANN-based network simulations can eliminate the need for expensive supercomputational resources and make large-scale network simulations more approachable for a wider audience.

\section{METHODS}

\section{Single-compartmental NEURON simulation}

Passive and active membrane responses to synaptic inputs were simulated in NEURON ( ${ }^{63}$, version 7.7, available at http://www.neuron.yale.edu/neuron/). Morphology (single compartment with length and diameter of $25 \mu \mathrm{m})$ and passive cellular parameters $\left(R_{m}: 1 \mathrm{k} \Omega / \mathrm{cm}^{2}, C_{m}: 1 \mu \mathrm{F} / \mathrm{cm}^{2}, R_{i}: 35.4 \Omega / \mathrm{cm}\right)$ were the 
same for both cases and resting membrane potential was set to $-70 \mathrm{mV}$. Additionally, the built-in mixed sodium, potassium and leak channel ( ${ }^{152}$, based on the original Hodgkin-Huxley descriptions) was included in the active model ( $\left.\mathrm{g}_{\mathrm{Na}}: 0.12 \mathrm{pS} / \mu \mathrm{m}^{2}, \mathrm{~g}_{\mathrm{K}}: 0.036 \mathrm{pS} / \mu \mathrm{m}^{2}, \mathrm{~g}_{\text {leak }}: 0.3 \mathrm{nS} / \mu \mathrm{m}^{2}\right)$. Reversal potentials were set to $50 \mathrm{mV}$ for sodium, $-77 \mathrm{mV}$ for potassium and $-54.3 \mathrm{mV}$ for leak conductance. Simulations were run with a custom steady state initialization procedure ${ }^{153}$ for 2 seconds, after which the temporal integration step size was set to $25 \mu \mathrm{s}$.

In order to simulate membrane responses to excitatory and inhibitory inputs, the built-in AlphaSynapse class of NEURON was used (excitatory synapse: $\tau: 2 \mathrm{~ms}, g_{\text {pas }}: 2.5 \mathrm{nS}, E_{\text {rev }}: 0 \mathrm{mV}$, inhibitory synapse: $\tau$ : $1 \mathrm{~ms}, g_{\text {pas }}: 8 \mathrm{nS}, E_{\text {rev }}:-90 \mathrm{mV}$ ). The number of synapses was determined by a pseudo-random uniform number generator (ratio of excitatory to inhibitory synapses: 8:3). Timing of individual synapses was also randomly picked from a uniform distribution. During the 10 -second-long simulations the membrane potential, $\mathrm{I}_{\mathrm{Na}}$ and $\mathrm{I}_{\mathrm{K}}$ currents were recorded along with the input timings and weights and were subsequently saved to text files. Simulations were carried out in three different conditions. First, resting membrane potential was recorded without synaptic activity. Second, passive membrane potential was recorded. Third, active membrane potential responses were recorded with fixed synaptic weights.

\section{Multicompartmental NEURON simulation}

Active multicompartmental simulations were carried out using an in vivo-labeled and fully reconstructed thick tufted cortical L5 PC ${ }^{43}$. The biophysical properties were unchanged and a class representation was created for network simulations. Excitatory and inhibitory synapses were handled similarly to singlecompartmental simulations. 100 excitatory ( $\left.\tau: 1 \mathrm{~ms}, g_{\text {pas }}: 3.6 \mathrm{nS}, E_{\text {rev }}: 0 \mathrm{mV}\right)$ and 30 inhibitory synapses $(\tau$. $1 \mathrm{~ms}, g_{\text {pas }}: 3 \mathrm{nS}, E_{\text {rev }}:-90 \mathrm{mV}$ ) were placed on the apical, oblique or tuft dendrites, 50 excitatory and 20 inhibitory synapses were placed on basal dendrites. The placement of the synapses was governed by two uniform pseudo-random number generators, which selected dendritic segments weighed by their respective lengths and the location along the segment (ratio: 2:1:1:1, for apical excitatory, apical inhibitory, basal excitatory and basal inhibitory synapses). Simulations were carried out with varied synaptic weights and a wide range of synapse numbers.

\section{ANN benchmarking}

To validate the overall suitability of different ANN architectures tested in this paper for multivariate time series forecasting, we used a weather time series dataset recorded by the Max Planck Institute for Biogeochemistry. The dataset contains 14 different features, including humidity, temperature and atmospheric pressure collected every 10 minutes. The dataset was prepared by François Chollet for his book Deep Learning with Python (dataset preparation steps can be found on the Tensorflow website: 
https://www.tensorflow.org/tutorials/structured_data/time_series). All ANN architectures were implemented using the Keras deep-learning API (https://keras.io/) of the Tensorflow open-source library (version 2.3, ${ }^{154}$, https://www.tensorflow.org/), with Python 3.7.

The first architecture we implemented was a simple linear model consisting of three layers without activation functions; a Flatten layer, a Dense (fully connected) layer with 64 units and a Dense layer with 3 units. The second architecture was a linear model with added nonlinear processing. The model contained three layers identical to the linear model, but the second layer had a sigmoid activation function. The third model was a deep neural net with mixed linear and nonlinear layers. Similar to the first two models, this architecture had a Flatten layer and a Dense layer with 64 units as the first two layers, followed by nine Dense layers (units: 128, 256, 512, 1024, 1024, 512, 256, 128, 64, for the 9 Dense layers) with hyperbolic tangent ( $\tanh$ ) activation function and Dropout layers with 0.15 dropout rate. The last layer was the same Dense layer with 3 units as in case of the linear and nonlinear models. The fourth model was a modified version of the WaveNet architecture introduced in $2016^{62}$, implemented based on a previous publication ${ }^{42}$. The fifth and final architecture was a convolutional LSTM model ${ }^{59}$ which consists of three distinct functional layer segments. The lowest layers (close to the input layer) were three, one dimensional convolutional layers (Conv1D) with 128, 100 and 50 units, and causal padding for temporal data processing. The first and third layer had kernel size of one and the second had kernel size of 5. The first two layers had "rectified linear unit" (relu) activation functions, and the third layer had tanh activation, therefore the first two layers were initialized by He-uniform variance scaling initializers ${ }^{87}$, while the third layer was initialized by Glorot-uniform initialization (also known as Xavier uniform initialization) ${ }^{155}$. After flattening and repeating the output of this functional unit, a single Long-Short Term Memory layer $\left(\mathrm{LSTM}^{60}\right)$ handled the arriving input, providing recurrent information processing. This layer had 128 units, tanh activation function, Glorot-uniform initialization and was tasked to return sequences instead of the last output. The final functional unit was composed of four Dense layers with 100 units, scaled exponential linear unit (selu) activations and accordingly, Lecun-uniform initializations ${ }^{156}$. The dropout rate between Dense layers was set to 0.15 .

All benchmarked architectures were compiled and fitted with the same protocol. During compiling, the loss function was set to calculate mean squared error and the Adam algorithm ${ }^{157}$ was chosen as the optimizer. The maximum number of epochs was set to 20 , however an early stopping protocol was defined to have a patience of 10 , which was reached in all cases.

\section{Single compartmental simulation representation with ANNs}

As neural nets favor processed data scaled between -1 and 1 or 0 and 1 , we normalized the recorded membrane potentials and ionic currents. Due to the $1 \mathrm{~Hz}$ recording frequency, AP amplitudes were variable 
beyond physiologically plausible ranges, therefore peak amplitudes were standardized. The trainable time series data was consisting of $64 \mathrm{~ms}$ long input matrices with 3 or 5 columns (corresponding to membrane potential, excitatory input, inhibitory input and optionally $\mathrm{I}_{\mathrm{Na}}$ and $\mathrm{I}_{\mathrm{K}}$ current recordings) and target sequences were vectors with 1 or 3 elements (membrane potential and optional ionic currents). Training, testing and validation datasets were created by splitting time series samples $80-10-10 \%$.

Benchmarking the five different ANN architectures proved that these models can handle time series data predicting with similar accuracy, however, in order to obtain the best results, several optimization steps of the hyperparameter space were undertaken. Unless it is stated otherwise, layer and optimization parameters were unchanged compared to benchmarking procedures. First, linear models were created without a Flatten layer, instead of which a TimeDistributed wrapper was applied to the first Dense layer. The same changes were employed in case of the nonlinear model and the deep neural net. The fourth, convolutional model had 12 Conv1D layers with 128 filters, kernel size of 2, causal padding tanh activation function and dilatation rates constantly increasing by $2^{\mathrm{n}}$. We found that the best optimization algorithm for passive and active membrane potential prediction is the Adam optimizer accelerated with Nesterov momentum ${ }^{158}$, with gradient clipping set to 1 . Although mean absolute error and mean absolute percentage error was sufficient for passive membrane potential prediction, the active version warranted the usage of mean squared error in order to put emphasis on APs. We found out that mechanistic inference of the full dynamic range of simulated neurons was a hard task for ANNs, therefore we sequentially trained these models in a specific order. First, we taught the resting membrane potential by supplying voltage recordings with only a few or no synaptic inputs. This step was also useful to learn the isolated shapes of certain inputs. Second, we supplied highly active subthreshold membrane traces to the models and finally we inputted suprathreshold membrane potential recordings. During the subsequent training steps, previous learning phases were mixed into the new training dataset in order to avoid the catastrophic forgetting of gradient based neural networks ${ }^{159}$.

\section{CCN-LSTM for multicompartmental simulation representation}

Data preprocessing was done as described for single compartmental representations. Time series data for CNN-LSTM input was prepared as matrices having 201 rows for membrane potential and 200 synapse vectors, and 64 rows (64 ms long input). The CNN-LSTM architecture consisted of three Conv1d layers (512, 256 and 128 units), a Flatten layer, a RepeatVector, three LSTM layers (128 units each) and six Dense layers $(128,100,100,100,100,1$ units). Activation functions and initializations were similar to the CNNLSTM described above, with the exception of the first Dense layer, which had relu activation function and He-uniform initialization. Additionally, Lasso regularization ${ }^{160}$ was applied to the first Conv1D layer. We found that the best optimizer for our purposes was a variant of the Adam optimizer based on the infinity 
norm, called Adamax ${ }^{157}$. Due to the non-normal distribution of the predicted membrane potentials, an inherent bias was present in our results, which was scaled by either an additional bias term, or a nonlinear function transformation.

Network construction was based on a previous publication ${ }^{98}$. Briefly, 150 L5 PC were simulated in a network with varying unidirectional connectivity, and bidirectional connectivity proportional to the unidirectional connectivity $\left(\mathrm{P}_{\text {bidirectional }}=0.5 * \mathrm{P}_{\text {unidirecional }}\right)$. Reciprocal connections were 1.5-times stronger than unidirectional connections. The delay between presynaptic AP at the soma and the onset of the postsynaptic response was $1 \mathrm{~ms}$ measured from the AP peak. Each connection consisted of five proximal contacts. Compared to the original publication, we modified the parameters of the Tsodyks-Markram mode $^{161}$ used to govern synaptic transmission and plasticity. Based on a recent publication ${ }^{103}$, we set $\mathrm{U}$ (fraction on synaptic resources used by a single spike) to 0.38 , D (time constant for recovery from depression) to 365.6 and $\mathrm{F}$ (time constant for recovery from facilitation) to 25.71 . The simulation was run for 250 or $300 \mathrm{~ms}$, which consisted of a pre-stimuli period (to observe the occurrence of structured activity patterns) for $100 \mathrm{~ms}$, and a post-stimuli period (to quantify network amplification). The stimulus itself consisted of a strong excitatory input (can be translated to $50 \mathrm{nS}$ ) delivered to a proximal dendritic segment, calibrated to elicit APs from all 150 cells in a $10 \mathrm{~ms}$ long time window. Scaling of inhibitory inputs was carried out by changing inhibitory quantal size of background inputs, while scaling of excitatory drive affected quantal size of recurrent synaptic connections as well.

\section{Computational resources}

We used several different commercially available and free-to-use computational resources to demonstrate the attainableness of large network simulations using neural networks. Single compartmental NEURON simulations were carried out on a CPU (Intel Core i7-5557U CPU @3.1 GHz). For multicompartmental NEURON simulations, we used the publicly available National Science Foundation funded High Performance Computing resource via the Neuroscience Gateway ${ }^{162}$. In contrast to NEURON models, ANN calculations are designed to run on GPUs rather than CPUs. Therefore, ANN models were run on the freely accessible Google Colaboratory GPUs (NVIDIA Tesla K80), Google Colaboratory tensor processing units (TPUs, designed for handling tensor calculations typically created by Tensorflow) and a single highperformance GPU (GeForce GTX 1080 Ti). For speed comparison we run these models on Google Colaboratory CPUs (Intel Xeon, not specified, @2.2 GHz) and the previously mentioned CPU as well. During NEURON and ANN simulations parallelization was only employed for Neuroscience Gateway simulations and ANN fitting.

\section{Statistics}


Averages of multiple measurements are presented as mean \pm SD. Data were statistically analyzed by ANOVA test using Origin software and custom written Python scripts. Normality of the data was analyzed with Shapira-Wilks test. Explained variance was quantified as one minus the fitting error normalized by the variance of the signal ${ }^{58}$. For accuracy measurements APs were counted within $10 \mathrm{~ms}$ time window as true positive APs. Precision and recall were calculated based on the following equations:

$$
\begin{gathered}
\text { precision }=\frac{T P}{T P+F P} \\
\text { recall }=\frac{T P}{T P+F N}
\end{gathered}
$$

where $\mathrm{FP}$ in the false positive rate and FN is the false negative rate.

\section{Data and software availability}

The code used for simulating single and multicompartmental NEURON models, ANN benchmarking, ANN representations and layer 5 microcircuit will be available upon publication.

\section{Acknowledgements}

Partial support (NPP) provided by CURE Epilepsy and the National Institutes of Health K08NS105929.

\section{REFERENCES}

1. Sejnowski TJ, Koch C, Churchland PSJS. Computational neuroscience. 1988;241(4871):1299-306.

2. Dayan P, Abbott LF. Theoretical neuroscience: computational and mathematical modeling of neural systems: Computational Neuroscience Series; 2001.

3. Kriegeskorte N, Douglas PKJNn. Cognitive computational neuroscience. 2018;21(9):1148-60.

4. Wolpert DM, Ghahramani ZJNn. Computational principles of movement neuroscience. 2000;3(11):1212-7.

5. Hodgkin AL, Huxley AFJTJop. A quantitative description of membrane current and its application to conduction and excitation in nerve. 1952;117(4):500-44.

6. Chavlis S, Petrantonakis PC, Poirazi PJH. Dendrites of dentate gyrus granule cells contribute to pattern separation by controlling sparsity. 2017;27(1):89-110.

7. Turi GF, Li W-K, Chavlis S, Pandi I, O'Hare J, Priestley JB, et al. Vasoactive intestinal polypeptideexpressing interneurons in the hippocampus support goal-oriented spatial learning. 2019;101(6):1150-65. e8.

8. Traub RD, Contreras D, Cunningham MO, Murray H, LeBeau FE, Roopun A, et al. Single-column thalamocortical network model exhibiting gamma oscillations, sleep spindles, and epileptogenic bursts. 2005;93(4):2194-232.

9. Neymotin SA, McDougal RA, Bulanova AS, Zeki M, Lakatos $P$, Terman D, et al. Calcium regulation of HCN channels supports persistent activity in a multiscale model of neocortex. 2016;316:344-66. 
10. Yu Y, McTavish TS, Hines ML, Shepherd GM, Valenti C, Migliore MJPCB. Sparse distributed representation of odors in a large-scale olfactory bulb circuit. 2013;9(3):e1003014.

11. Migliore M, Hoffman DA, Magee JC, Johnston DJJocn. Role of an A-type K+ conductance in the back-propagation of action potentials in the dendrites of hippocampal pyramidal neurons. 1999;7(1):515.

12. Migliore $M$, Cook E, Jaffe $D$, Turner $D$, Johnston DJJon. Computer simulations of morphologically reconstructed CA3 hippocampal neurons. 1995;73(3):1157-68.

13. De Schutter E, Bower JMJJon. An active membrane model of the cerebellar Purkinje cell. I. Simulation of current clamps in slice. 1994;71(1):375-400.

14. Aradi I, Holmes WRJJocn. Role of multiple calcium and calcium-dependent conductances in regulation of hippocampal dentate granule cell excitability. 1999;6(3):215-35.

15. Mainen ZF, Sejnowski TJJN. Influence of dendritic structure on firing pattern in model neocortical neurons. 1996;382(6589):363-6.

16. Destexhe A, Neubig M, Ulrich D, Huguenard JJJoN. Dendritic low-threshold calcium currents in thalamic relay cells. 1998;18(10):3574-88.

17. Stuart G, Spruston NJJoN. Determinants of voltage attenuation in neocortical pyramidal neuron dendrites. 1998;18(10):3501-10.

18. Mainen ZF, Joerges J, Huguenard JR, Sejnowski TJJN. A model of spike initiation in neocortical pyramidal neurons. 1995;15(6):1427-39.

19. Bezaire MJ, Raikov I, Burk K, Vyas D, Soltesz IJE. Interneuronal mechanisms of hippocampal theta oscillations in a full-scale model of the rodent CA1 circuit. 2016;5:e18566.

20. Markram H, Muller E, Ramaswamy S, Reimann MW, Abdellah M, Sanchez CA, et al. Reconstruction and simulation of neocortical microcircuitry. 2015;163(2):456-92.

21. Billeh YN, Cai B, Gratiy SL, Dai K, Iyer R, Gouwens NW, et al. Systematic integration of structural and functional data into multi-scale models of mouse primary visual cortex. 2020;106(3):388-403. e18.

22. Arkhipov A, Gouwens NW, Billeh YN, Gratiy S, lyer R, Wei Z, et al. Visual physiology of the layer 4 cortical circuit in silico. 2018;14(11):e1006535.

23. Joglekar MR, Mejias JF, Yang GR, Wang X-JJN. Inter-areal balanced amplification enhances signal propagation in a large-scale circuit model of the primate cortex. 2018;98(1):222-34. e8.

24. Schmidt M, Bakker R, Hilgetag CC, Diesmann M, van Albada SJJBS, Function. Multi-scale account of the network structure of macaque visual cortex. 2018;223(3):1409-35.

25. Antolík J, Monier C, Frégnac Y, Davison APJB. A comprehensive data-driven model of cat primary visual cortex. 2019:416156.

26. Schwalger T, Chizhov AVJCoin. Mind the last spike-firing rate models for mesoscopic populations of spiking neurons. 2019;58:155-66.

27. Wang X-J, Buzsáki GJJon. Gamma oscillation by synaptic inhibition in a hippocampal interneuronal network model. 1996;16(20):6402-13.

28. Bartos M, Vida I, Frotscher M, Meyer A, Monyer H, Geiger JR, et al. Fast synaptic inhibition promotes synchronized gamma oscillations in hippocampal interneuron networks. 2002;99(20):13222-7.

29. Cutsuridis V, Cobb S, Graham BPJH. Encoding and retrieval in a model of the hippocampal CA1 microcircuit. 2010;20(3):423-46.

30. Santhakumar V, Aradi I, Soltesz IJJon. Role of mossy fiber sprouting and mossy cell loss in hyperexcitability: a network model of the dentate gyrus incorporating cell types and axonal topography. 2005;93(1):437-53.

31. Teeter C, lyer R, Menon V, Gouwens N, Feng D, Berg J, et al. Generalized leaky integrate-and-fire models classify multiple neuron types. 2018;9(1):1-15.

32. Wybo WA, Jordan J, Ellenberger B, Mengual UM, Nevian T, Senn WJE. Data-driven reduction of dendritic morphologies with preserved dendro-somatic responses. 2021;10:e60936. 
33. Amsalem O, Eyal G, Rogozinski N, Gevaert M, Kumbhar P, Schürmann F, et al. An efficient analytical reduction of detailed nonlinear neuron models. 2020;11(1):1-13.

34. Merembayev T, Yunussov R, Yedilkhan A, editors. Machine learning algorithms for classification geology data from well logging. 2018 14th International Conference on Electronics Computer and Computation (ICECCO); 2018: IEEE.

35. Montavon G, Rupp M, Gobre V, Vazquez-Mayagoitia A, Hansen K, Tkatchenko A, et al. Machine learning of molecular electronic properties in chemical compound space. 2013;15(9):095003.

36. Meredig B, Agrawal A, Kirklin S, Saal JE, Doak J, Thompson A, et al. Combinatorial screening for new materials in unconstrained composition space with machine learning. 2014;89(9):094104.

37. Schütt KT, Chmiela S, von Lilienfeld OA, Tkatchenko A, Tsuda K, Müller K-R, et al. Machine Learning Meets Quantum Physics: Springer; 2020.

38. Sharma N, Sharma $P$, Irwin D, Shenoy $P$, editors. Predicting solar generation from weather forecasts using machine learning. 2011 IEEE international conference on smart grid communications (SmartGridComm); 2011: IEEE.

39. Holmstrom M, Liu D, Vo CJMA. Machine learning applied to weather forecasting. 2016.

40. Ghoddusi H, Creamer GG, Rafizadeh NJEE. Machine learning in energy economics and finance: A review. 2019;81:709-27.

41. Poirazi P, Brannon T, Mel BWJN. Pyramidal neuron as two-layer neural network. 2003;37(6):989-

99.

42. Beniaguev D, Segev I, London MJb. Single cortical neurons as deep artificial neural networks. 2020:613141.

43. Hallermann S, De Kock CP, Stuart GJ, Kole MHJNn. State and location dependence of action potential metabolic cost in cortical pyramidal neurons. 2012;15(7):1007-14.

44. Hagberg B, Goutières F, Hanefeld F, Rett A, Wilson JJB, development. Rett syndrome: criteria for inclusion and exclusion. 1985;7(3):372-3.

45. Chahrour M, Zoghbi HYJN. The story of Rett syndrome: from clinic to neurobiology. 2007;56(3):422-37.

46. Duncan Armstrong DJJocn. Neuropathology of Rett syndrome. 2005;20(9):747-53.

47. Glaze DGJJocn. Neurophysiology of Rett syndrome. 2005;20(9):740-6.

48. Kons Z, Toledo-Ronen $\mathrm{O}$, Carmel $\mathrm{M}$, editors. Audio event classification using deep neural networks. Interspeech; 2013.

49. Collobert R, Weston J, editors. A unified architecture for natural language processing: Deep neural networks with multitask learning. Proceedings of the 25th international conference on Machine learning; 2008.

50. Zheng Y, Liu Q, Chen E, Ge Y, Zhao JL, editors. Time series classification using multi-channels deep convolutional neural networks. International conference on web-age information management; 2014: Springer.

51. Che Z, Purushotham S, Cho K, Sontag D, Liu Y. Recurrent neural networks for multivariate time series with missing values. Scientific reports. 2018;8(1):1-12.

52. Zhang C, Song D, Chen Y, Feng X, Lumezanu C, Cheng W, et al., editors. A deep neural network for unsupervised anomaly detection and diagnosis in multivariate time series data. Proceedings of the AAAI Conference on Artificial Intelligence; 2019.

53. Yang GR, Wang X-J. Artificial neural networks for neuroscientists: A primer. Neuron. 2020;107(6):1048-70.

54. Alwosheel A, van Cranenburgh S, Chorus CGJJocm. Is your dataset big enough? Sample size requirements when using artificial neural networks for discrete choice analysis. 2018;28:167-82.

55. Jaitly N, Nguyen P, Senior A, Vanhoucke V. Application of pretrained deep neural networks to large vocabulary speech recognition. 2012. 
56. Jia F, Lei Y, Lin J, Zhou X, Lu NJMS, Processing S. Deep neural networks: A promising tool for fault characteristic mining and intelligent diagnosis of rotating machinery with massive data. 2016;72:303-15.

57. Abello J, Pardalos PM, Resende MG. Handbook of massive data sets: Springer; 2013.

58. Ujfalussy BB, Makara JK, Lengyel M, Branco TJN. Global and multiplexed dendritic computations under in vivo-like conditions. 2018;100(3):579-92. e5.

59. Donahue J, Anne Hendricks L, Guadarrama S, Rohrbach M, Venugopalan S, Saenko K, et al., editors. Long-term recurrent convolutional networks for visual recognition and description. Proceedings of the IEEE conference on computer vision and pattern recognition; 2015.

60. Hochreiter S, Schmidhuber JJNc. Long short-term memory. 1997;9(8):1735-80.

61. Shi X, Chen Z, Wang H, Yeung D-Y, Wong W-K, Woo W-cJapa. Convolutional LSTM network: A machine learning approach for precipitation nowcasting. 2015.

62. Oord Avd, Dieleman S, Zen H, Simonyan K, Vinyals O, Graves A, et al. Wavenet: A generative model for raw audio. 2016.

63. Hines ML, Carnevale NTJNc. The NEURON simulation environment. 1997;9(6):1179-209.

64. Rumelhart DE, Hinton GE, Williams RJJn. Learning representations by back-propagating errors. 1986;323(6088):533-6.

65. Kawaguchi KJapa. Deep learning without poor local minima. 2016.

66. Graupe D. Principles of artificial neural networks: World Scientific; 2013.

67. Hassoun MH. Fundamentals of artificial neural networks: MIT press; 1995.

68. Magee JC, Cook EPJNn. Somatic EPSP amplitude is independent of synapse location in hippocampal pyramidal neurons. 2000;3(9):895-903.

69. Stuart G, Spruston N, Häusser M. Dendrites: Oxford University Press; 2016.

70. Stuart GJ, Spruston NJNn. Dendritic integration: 60 years of progress. 2015;18(12):1713-21.

71. Kole MH, Ilschner SU, Kampa BM, Williams SR, Ruben PC, Stuart GJJNn. Action potential generation requires a high sodium channel density in the axon initial segment. 2008;11(2):178-86.

72. Kim S, Guzman SJ, Hu H, Jonas PJNn. Active dendrites support efficient initiation of dendritic spikes in hippocampal CA3 pyramidal neurons. 2012;15(4):600-6.

73. Rowan MJ, Tranquil E, Christie JMJJoN. Distinct Kv channel subtypes contribute to differences in spike signaling properties in the axon initial segment and presynaptic boutons of cerebellar interneurons. 2014;34(19):6611-23.

74. Brunner J, Szabadics JJNc. Analogue modulation of back-propagating action potentials enables dendritic hybrid signalling. 2016;7(1):1-13.

75. Losonczy A, Makara JK, Magee JCJN. Compartmentalized dendritic plasticity and input feature storage in neurons. 2008;452(7186):436-41.

76. Major G, Larkman AU, Jonas P, Sakmann B, Jack JJJoN. Detailed passive cable models of wholecell recorded CA3 pyramidal neurons in rat hippocampal slices. 1994;14(8):4613-38.

77. Vetter $P$, Roth $A$, Häusser MJJon. Propagation of action potentials in dendrites depends on dendritic morphology. 2001;85(2):926-37.

78. Oláh VJ, Lukacsovich D, Winterer J, Arszovszki A, Lőrincz A, Nusser Z, et al. Functional specification of CCK+ interneurons by alternative isoforms of Kv4. 3 auxiliary subunits. 2020;9:e58515.

79. Häusser M, Mel BJCoin. Dendrites: bug or feature? 2003;13(3):372-83.

80. Takahashi N, Oertner TG, Hegemann P, Larkum MEJS. Active cortical dendrites modulate perception. 2016;354(6319):1587-90.

81. Jarsky T, Roxin A, Kath WL, Spruston NJNn. Conditional dendritic spike propagation following distal synaptic activation of hippocampal CA1 pyramidal neurons. 2005;8(12):1667-76.

82. Schiller J, Schiller Y, Stuart G, Sakmann BJTJop. Calcium action potentials restricted to distal apical dendrites of rat neocortical pyramidal neurons. 1997;505(3):605-16. 
83. Harnett MT, Magee JC, Williams SRJJoN. Distribution and function of HCN channels in the apical dendritic tuft of neocortical pyramidal neurons. 2015;35(3):1024-37.

84. Larkum ME, Nevian T, Sandler M, Polsky A, Schiller JJS. Synaptic integration in tuft dendrites of layer 5 pyramidal neurons: a new unifying principle. 2009;325(5941):756-60.

85. Takahashi $\mathrm{H}$, Magee JCJN. Pathway interactions and synaptic plasticity in the dendritic tuft regions of CA1 pyramidal neurons. 2009;62(1):102-11.

86. Sjöström PJ, Häusser MJN. A cooperative switch determines the sign of synaptic plasticity in distal dendrites of neocortical pyramidal neurons. 2006;51(2):227-38.

87. He K, Zhang X, Ren S, Sun J, editors. Delving deep into rectifiers: Surpassing human-level performance on imagenet classification. Proceedings of the IEEE international conference on computer vision; 2015.

88. Destexhe A, Sejnowski TJ. Thalamocortical Assemblies: How lon Channels, Single Neurons and Large-Scale Networks Organize Sleep Oscillations: Oxford University Press, USA; 2001.

89. Hendrickson EB, Edgerton JR, Jaeger DJJocn. The capabilities and limitations of conductancebased compartmental neuron models with reduced branched or unbranched morphologies and active dendrites. 2011;30(2):301-21.

90. Bush PC, Sejnowski TJJJonm. Reduced compartmental models of neocortical pyramidal cells. 1993;46(2):159-66.

91. Marasco A, Limongiello A, Migliore MJSr. Fast and accurate low-dimensional reduction of biophysically detailed neuron models. 2012;2(1):1-7.

92. Rössert C, Pozzorini C, Chindemi G, Davison AP, Eroe C, King J, et al. Automated point-neuron simplification of data-driven microcircuit models. 2016.

93. Hines ML, Markram H, Schürmann FJJocn. Fully implicit parallel simulation of single neurons. 2008;25(3):439-48.

94. Hines ML, Carnevale NTJJonm. Translating network models to parallel hardware in NEURON. 2008;169(2):425.

95. Lytton WW, Seidenstein AH, Dura-Bernal S, McDougal RA, Schürmann F, Hines MLNc. Simulation neurotechnologies for advancing brain research: parallelizing large networks in NEURON. 2016;28(10):2063-90.

96. Cutsuridis V, Wennekers TJNN. Hippocampus, microcircuits and associative memory. 2009;22(8):1120-8.

97. Nikolic D, Haeusler S, Singer W, Maass W, editors. Temporal dynamics of information content carried by neurons in the primary visual cortex. NIPS; 2006.

98. Hay E, Segev IJCC. Dendritic excitability and gain control in recurrent cortical microcircuits. 2015;25(10):3561-71.

99. Chadderdon GL, Mohan A, Suter BA, Neymotin SA, Kerr CC, Francis JT, et al. Motor cortex microcircuit simulation based on brain activity mapping. 2014;26(7):1239-62.

100. Migliore M, Shepherd GMJJocn. Dendritic action potentials connect distributed dendrodendritic microcircuits. 2008;24(2):207-21.

101. Shimoura RO, Kamiji NL, de Oliveira Pena RF, Cordeiro VL, Ceballos CC, Romaro C, et al. Reimplementation of the Potjans-Diesmann cortical microcircuit model: from NEST to Brian. 2018:248401.

102. Kumbhar P, Hines M, Fouriaux J, Ovcharenko A, King J, Delalondre F, et al. CoreNEURON: an optimized compute engine for the NEURON simulator. 2019;13:63.

103. Barros-Zulaica N, Rahmon J, Chindemi G, Perin R, Markram H, Muller E, et al. Estimating the readily-releasable vesicle pool size at synaptic connections in the neocortex. 2019;11:29.

104. Belichenko PV, Oldfors A, Hagberg B, Dahlström AJNAIJftRCoRiN. Rett syndrome: 3-D confocal microscopy of cortical pyramidal dendrites and afferents. 1994. 
105. Belichenko PV, Wright EE, Belichenko NP, Masliah E, Li HH, Mobley WC, et al. Widespread changes in dendritic and axonal morphology in Mecp2-mutant mouse models of rett syndrome: Evidence for disruption of neuronal networks. 2009;514(3):240-58.

106. Armstrong DDJMr, reviews ddr. Neuropathology of Rett syndrome. 2002;8(2):72-6.

107. Kishi N, Macklis JDJM, Neuroscience C. MECP2 is progressively expressed in post-migratory neurons and is involved in neuronal maturation rather than cell fate decisions. 2004;27(3):306-21.

108. Fukuda T, Yamashita Y, Nagamitsu S, Miyamoto K, Jin J-J, Ohmori I, et al. Methyl-CpG binding protein 2 gene (MECP2) variations in Japanese patients with Rett syndrome: pathological mutations and polymorphisms. 2005;27(3):211-7.

109. Armstrong DDJJoN, Neurology E. Review of Rett syndrome. 1997;56(8):843-9.

110. Steffenburg U, Hagberg G, Hagberg BJAp. Epilepsy in a representative series of Rett syndrome. 2001;90(1):34-9.

111. Dani VS, Chang Q, Maffei A, Turrigiano GG, Jaenisch R, Nelson SBJPotNAoS. Reduced cortical activity due to a shift in the balance between excitation and inhibition in a mouse model of Rett syndrome. 2005;102(35):12560-5.

112. Medrihan L, Tantalaki E, Aramuni G, Sargsyan V, Dudanova I, Missler M, et al. Early defects of GABAergic synapses in the brain stem of a MeCP2 mouse model of Rett syndrome. 2008;99(1):112-21.

113. Zhang Z-W, Zak JD, Liu HJJon. MeCP2 is required for normal development of GABAergic circuits in the thalamus. 2010;103(5):2470-81.

114. Akbarian S, Chen RZ, Gribnau J, Rasmussen TP, Fong H-f, Jaenisch R, et al. Expression pattern of the Rett syndrome gene MeCP2 in primate prefrontal cortex. 2001;8(5):784-91.

115. Dani VS, Nelson SBJJoN. Intact long-term potentiation but reduced connectivity between neocortical layer 5 pyramidal neurons in a mouse model of Rett syndrome. 2009;29(36):11263-70.

116. De Kock C, Bruno RM, Spors H, Sakmann BJTJop. Layer-and cell-type-specific suprathreshold stimulus representation in rat primary somatosensory cortex. 2007;581(1):139-54.

117. Meyer HS, Wimmer VC, Hemberger M, Bruno RM, de Kock CP, Frick A, et al. Cell type-specific thalamic innervation in a column of rat vibrissal cortex. 2010;20(10):2287-303.

118. Constantinople CM, Bruno RMJS. Deep cortical layers are activated directly by thalamus. 2013;340(6140):1591-4.

119. Sun YJ, Kim Y-J, Ibrahim LA, Tao HW, Zhang LIJJoN. Synaptic mechanisms underlying functional dichotomy between intrinsic-bursting and regular-spiking neurons in auditory cortical layer 5. 2013;33(12):5326-39.

120. Lien AD, Scanziani MJNn. Tuned thalamic excitation is amplified by visual cortical circuits. 2013;16(9):1315-23.

121. McCormick DA, Contreras DJArop. On the cellular and network bases of epileptic seizures. 2001;63(1):815-46.

122. Roche KJ, LeBlanc JJ, Levin AR, O'Leary HM, Baczewski LM, Nelson CAJJond. Electroencephalographic spectral power as a marker of cortical function and disease severity in girls with Rett syndrome. 2019;11(1):1-14.

123. McLeod F, Ganley R, Williams L, Selfridge J, Bird A, Cobb SJN. Reduced seizure threshold and altered network oscillatory properties in a mouse model of Rett syndrome. 2013;231:195-205.

124. D'Cruz JA, Wu C, Zahid T, El-Hayek Y, Zhang L, Eubanks JHJNod. Alterations of cortical and hippocampal EEG activity in MeCP2-deficient mice. 2010;38(1):8-16.

125. Chao H-T, Chen H, Samaco RC, Xue M, Chahrour M, Yoo J, et al. Dysfunction in GABA signalling mediates autism-like stereotypies and Rett syndrome phenotypes. 2010;468(7321):263-9.

126. Benítez JM, Castro JL, Requena IJITonn. Are artificial neural networks black boxes? 1997;8(5):1156-64. 
127. Dayhoff JE, DeLeo JMJCIIJotACS. Artificial neural networks: opening the black box. 2001;91(S8):1615-35.

128. Castelvecchi DJNN. Can we open the black box of Al? 2016;538(7623):20.

129. Da Silva IN, Spatti DH, Flauzino RA, Liboni LHB, dos Reis Alves SF. Artificial neural network architectures and training processes. Artificial neural networks: Springer; 2017. p. 21-8.

130. Zhang X, Santaniello SJPotNAoS. Role of cerebellar GABAergic dysfunctions in the origins of essential tremor. 2019;116(27):13592-601.

131. Liou J-y, Smith EH, Bateman LM, Bruce SL, McKhann GM, Goodman RR, et al. A model for focal seizure onset, propagation, evolution, and progression. 2020;9:e50927.

132. Sanjay M, Neymotin SA, Krothapalli SB, Lytton WWJCMoB, Behavior. Multiscale Computer Modeling of Epilepsy. 2017:139.

133. Kerr C, Van Albada S, Neymotin S, Chadderdon G, Robinson P, Lytton W. Cortical information flow in Parkinson's disease: a composite network/field model. 2013;7(39).

134. Domanski AP, Booker SA, Wyllie DJ, Isaac JT, Kind PCJNc. Cellular and synaptic phenotypes lead to disrupted information processing in Fmr1-KO mouse layer 4 barrel cortex. 2019;10(1):1-18.

135. Gambazzi L, Gokce O, Seredenina T, Katsyuba E, Runne H, Markram H, et al. Diminished ActivityDependent Brain-Derived Neurotrophic Factor Expression Underlies Cortical Neuron Microcircuit Hypoconnectivity Resulting from Exposure to Mutant Huntingtin Fragments. 2010;335(1):13-22.

136. Neymotin SA, Dura-Bernal S, Lakatos P, Sanger TD, Lytton WW. Multitarget Multiscale Simulation for Pharmacological Treatment of Dystonia in Motor Cortex. 2016;7(157).

137. Dillon JV, Langmore I, Tran D, Brevdo E, Vasudevan S, Moore D, et al. Tensorflow distributions. 2017.

138. Memon ZA, Samad F, Awan ZR, Aziz A, Siddiqi SSJIJCSNS. Cpu-gpu processing. 2017;17(9):188-93. 139. Asano S, Maruyama T, Yamaguchi Y, editors. Performance comparison of FPGA, GPU and CPU in image processing. 2009 international conference on field programmable logic and applications; 2009: IEEE.

140. Vooturi DT, Kothapalli K, Bhalla US, editors. Parallelizing Hines matrix solver in neuron simulations on GPU. 2017 IEEE 24th International Conference on High Performance Computing (HiPC); 2017: IEEE.

141. Nelson ED, Kavalali ET, Monteggia LMJCB. MeCP2-dependent transcriptional repression regulates excitatory neurotransmission. 2006;16(7):710-6.

142. Chao H-T, Zoghbi HY, Rosenmund CJN. MeCP2 controls excitatory synaptic strength by regulating glutamatergic synapse number. 2007;56(1):58-65.

143. Na ES, Nelson ED, Adachi M, Autry AE, Mahgoub MA, Kavalali ET, et al. A mouse model for MeCP2 duplication syndrome: MeCP2 overexpression impairs learning and memory and synaptic transmission. 2012;32(9):3109-17.

144. Asaka Y, Jugloff DG, Zhang L, Eubanks JH, Fitzsimonds RMJNod. Hippocampal synaptic plasticity is impaired in the Mecp2-null mouse model of Rett syndrome. 2006;21(1):217-27.

145. Guy J, Gan J, Selfridge J, Cobb S, Bird AJS. Reversal of neurological defects in a mouse model of Rett syndrome. 2007;315(5815):1143-7.

146. Calfa G, Hablitz JJ, Pozzo-Miller LJon. Network hyperexcitability in hippocampal slices from Mecp2 mutant mice revealed by voltage-sensitive dye imaging. 2011;105(4):1768-84.

147. Zhang W, Peterson M, Beyer B, Frankel WN, Zhang Z-wJJoN. Loss of MeCP2 from forebrain excitatory neurons leads to cortical hyperexcitation and seizures. 2014;34(7):2754-63.

148. Behnel S, Bradshaw R, Citro C, Dalcin L, Seljebotn DS, Smith KJCiS, et al. Cython: The best of both worlds. 2010;13(2):31-9.

149. Lam SK, Pitrou A, Seibert S, editors. Numba: A llvm-based python jit compiler. Proceedings of the Second Workshop on the LLVM Compiler Infrastructure in HPC; 2015. 
150. Tan C, Sun F, Kong T, Zhang W, Yang C, Liu C, editors. A survey on deep transfer learning. International conference on artificial neural networks; 2018: Springer.

151. Lu J, Behbood V, Hao P, Zuo H, Xue S, Zhang GJK-BS. Transfer learning using computational intelligence: A survey. 2015;80:14-23.

152. Jaslove SJN. The integrative properties of spiny distal dendrites. 1992;47(3):495-519.

153. Carnevale NT, Hines ML. The NEURON book: Cambridge University Press; 2006.

154. Abadi M, Agarwal A, Barham P, Brevdo E, Chen Z, Citro C, et al. TensorFlow: Large-scale machine learning on heterogeneous systems. 2015.

155. Glorot X, Bordes A, Bengio Y, editors. Deep sparse rectifier neural networks. Proceedings of the fourteenth international conference on artificial intelligence and statistics; 2011: JMLR Workshop and Conference Proceedings.

156. LeCun YA, Bottou L, Orr GB, Müller K-R. Efficient backprop. Neural networks: Tricks of the trade: Springer; 2012. p. 9-48.

157. Kingma DP, Ba JJapa. Adam: A method for stochastic optimization. 2014.

158. Dozat T. Incorporating Nesterov momentum into Adam Technical Report. Stanford, CA: Stanford University; 2015.

159. Goodfellow IJ, Mirza M, Xiao D, Courville A, Bengio YJapa. An empirical investigation of catastrophic forgetting in gradient-based neural networks (2013). 2015.

160. Santosa F, Symes WWJSJoS, Computing S. Linear inversion of band-limited reflection seismograms. 1986;7(4):1307-30.

161. Tsodyks MV, Markram HJPotnaos. The neural code between neocortical pyramidal neurons depends on neurotransmitter release probability. 1997;94(2):719-23.

162. Sivagnanam S, Majumdar A, Yoshimoto K, Astakhov V, Bandrowski AE, Martone ME, et al. Introducing the Neuroscience Gateway. 2013;993. 